\title{
Topographic rectification in a forced, dissipative, barotropic ocean
}

\author{
by K. H. Brink ${ }^{1}$
}

\begin{abstract}
Barotropic current rectification by topographic irregularities is treated for a case with bottom friction and fluctuating forcing. Geometries both with underlying shelf-slope topography and with no mean topographic slope are considered. In common with many previous studies of this sort, the resulting time-mean flow roughly follows isobaths in the direction that long topographic Rossby waves travel, but the mean flow often deviates locally from this rule. Further, as might be expected, there is an area-averaged correlation of pressure and bottom slope in the sense that would propel the mean flow. If the topographic irregularities have a length scale shorter than roughly a particle fluctuation excursion, then the typical along-isobath mean flow is proportional to the bottom slope, the irregularity length scale, the amplitude of the cross-isobath velocity fluctuations, and the inverse of the water depth. If the spatial scale of the irregularities is greater than roughly a particle excursion, then the resulting mean flow does not depend on irregularity length scale, but does depend on the Coriolis parameter, the bottom slope, cross-isobath velocity squared, the inverse depth and the inverse frequency squared. For large amplitude fluctuations, eddy momentum transport leads to a further inverse proportionality of mean flow to the strength of bottom friction. The overall mean flow parameterization holds only in a statistical sense (as opposed to point-by-point) because of the spatial complexity of typical flows. In a forced, dissipative system, the mean flow generation is often just tidal rectification (e.g., Loder, 1980) if the particle excursion is short relative to topographic scales. However, as the irregularity scale decreases, mean flow becomes weaker.
\end{abstract}

\section{Introduction}

Numerous publications have developed the idea that zero-mean eddying flow fields naturally evolve toward a state where the mean flow approximately follows the isobaths in the direction of long topographic Rossby wave propagation. Initially, these ideas were expressed in terms of adiabatic enstrophy minimization (Bretherton and Haidvogel, 1976), nonlinear stability (Bretherton and Haidvogel, 1976; LaCasce et al., 2008) or of entropy maximization (Salmon et al., 1976), but these approaches have been shown to be largely equivalent (Carnevale and Frederiksen, 1987). These ideas have since been expanded to include the effects of dissipation (Herring, 1977; Holloway, 1978), systematic bottom slopes (Holloway, 1987), finite-amplitude topography (Merryfield et al., 2001), or simple

1. Department of Physical Oceanography, Woods Hole Oceanographic Institution, Woods Hole, Massachusetts, 02543, U.S.A.email: kbrink@whoi.edu 
stratification (Merryfield, 1998). Others have dealt less statistically with idealized dissipative, forced systems (e.g., Haidvogel and Brink, 1986 - HB hereafter; Samelson and Allen, 1987; Treguier and McWilliams, 1990; Alvarez et al., 1997), and have shown that topographic rectification can occur in these circumstances, although none of these latter publications developed a parameterization for the resulting dissipative mean flow.

Some evidence supports the presence of topographic rectification in the ocean. One approach has been to show that including a nudging term that drives a model along-isobath flow does indeed improve agreement between measurements and numerical model calculations (e.g., Holloway and Sou, 1996). This approach has been bedeviled because the predicted flow amplitude is governed by a free constant that is not well constrained. A second approach (Holloway, 2008) has been to use current meter data to show that, on a global average, mean ocean flows follow isobaths in the expected sense much more frequently than might be expected by chance. The problem with this approach is that, while it is impressive as a collective result, for any given location, one might be able to rationalize the mean flow direction by means other than topographic rectification.

The idea of the present study is to use well-resolved forced, dissipative barotropic numerical models to treat topographic rectification in an idealized form. The model outputs are then used to motivate and evaluate a scaling for mean flow in this system. The intent here is to provide expressions for how strong the topographically rectified flow might actually be in a given ocean setting, and to use the scaling to elucidate the underlying physics.

Many of the central concepts here arise from the rich and diverse tidal rectification literature. One thread (e.g., Huthnance, 1973; Stern and Shen, 1976) has considered cross-isobath momentum fluxes that develop in tidal oscillations with bottom friction. These fluxes accumulate mean along-isobath momentum and are balanced by the bottom drag on the mean flow. This general approach has been expanded analytically to treat nonlinearities (e.g., Loder, 1980), vertical structure (e.g., Wright and Loder, 1985), and density stratification in the limit of small bottom relief (Maas and Zimmerman, 1989). A second approach to tidal rectification has dealt with the tendency for an inviscid problem to allow a finite-amplitude fluctuating flow to redistribute momentum across isobaths (e.g., Robinson, 1981; Maas et al., 1987). This concept has since been developed to show that the resulting mean flow has a similar parametric dependence (e.g., on bottom slope, fluctuation amplitude, and rotation) to that of the frictional case (Garreau and Maze, 1992; Visser, 1994). Numerical models of tidal rectification naturally include both the frictional and finite amplitude aspects of rectification, and frequently also include density stratification as well (Chen and Beardsley, 1995; Perenne et al., 2000). Along-isobath variability has been included in many numerical models, and was treated analytically by Zimmerman (1978, 1980), who considered small amplitude bumps in the presence of a relatively spatially uniform fluctuating flow. There does not appear to have been much consideration to date of idealized flows with simple variability and finite-amplitude topography; i.e., a twodimensional equivalent to Loder's (1980) problem. 


\section{Model configuration}

The barotropic, depth-averaged, hydrostatic equations of motion (in the absence of lateral dissipation) are

$$
\begin{aligned}
& u_{t}+u u_{x}+v u_{y}-f v=-\rho^{-1} p_{x}-r u / h \\
& v_{t}+u v_{x}+v v_{y}+f u=-\rho^{-1} p_{y}-r v / h+\rho^{-1} \tau / h, \\
& (h u)_{x}+(h v)_{y}=0,
\end{aligned}
$$

where $(u, v)$ are the offshore $(x)$ and northward $(y)$ depth-averaged velocity, $\rho$ is the constant water density, $p$ is pressure, $h(x, y)$ is the water depth, $f$ is the (constant) Coriolis parameter, $\tau$ is the alongshore component of wind stress, and $r$ is a constant resistance coefficient associated with a linearized bottom stress. Subscripts with regard to independent variables represent partial differentiation. For purposes of this analysis, a rigid lid is assumed. Henceforth, to avoid geographical bias, the direction of long topographic Rossby wave propagation (southward in this geometry) will be called "downwave" and the opposing direction "upwave."

All model runs use the ROMS (Shchepetkin and McWilliams, 2005) primitive equation numerical model in its barotropic form (which uses 3 grid points in the vertical, has constant density and treats boundary stresses as body forces). Although the model uses a free-surface boundary condition, its effect at the scales of interest here is negligible. All model runs take place in a channel which is cyclic in the $y$ direction and has a wall along its coastal boundary $(x=0)$. Offshore, a radiative open boundary condition is imposed on sea-surface height. This offshore condition does not represent a significant energy sink: averaged over the last full forcing cycle, the total energy flux through this boundary is always 4-8 orders of magnitude smaller than the frictional dissipation within the domain. The alongshore momentum flux through this boundary is similarly miniscule. For all two-slope model runs (Section 3), resolution is $1 \mathrm{~km}$ in $x$ and either $1.5 \mathrm{~km}$ or (for the runs flagged with "F" in Table 1) $0.5 \mathrm{~km}$ in the alongshore direction. No horizontal diffusivity or viscosity is used. For the large-domain runs (Section 5), the resolution is $1 \mathrm{~km}$ in both directions. Either model is forced by an oscillating alongshore $(y)$ wind stress that is spatially uniform unless otherwise noted. All model runs reported here are carried out for at least 64 model days, which allows initial transients to decay completely in most cases. If a quasi-steady (meaning repeatable) or clearly chaotic state is not reached in 64 days, the model is run for up to 288 days until a quasi-steady state is reached. The model runs are summarized in Tables 1 and 2.

The model is forced by an alongshore wind stress,

$$
\tau=\sum_{q} d_{q} \sin \left(\omega_{q} t\right)
$$


Table 1. Tabulation of two-slope topography model runs.

\begin{tabular}{|c|c|c|c|c|c|c|c|c|c|c|}
\hline $\begin{array}{c}\text { Run } \\
\text { number }\end{array}$ & $f(1 / \mathrm{sec})$ & $r(\mathrm{~m} / \mathrm{sec})$ & $\begin{array}{l}h_{M} \\
(\mathrm{~m})\end{array}$ & $\lambda(\mathrm{km})$ & $\begin{array}{l}h_{C} \\
(\mathrm{~m})\end{array}$ & $\begin{array}{c}T \\
\text { (days) }\end{array}$ & $\begin{array}{c}d_{1} \\
\left(\mathrm{~N} / \mathrm{m}^{2}\right)\end{array}$ & $\Xi$ & Mult. & $\begin{array}{l}\bar{v}_{\|} \operatorname{Min} \\
(\mathrm{m} / \mathrm{sec})\end{array}$ \\
\hline 300 & $1 \times 10^{-4}$ & $5 \times 10^{-4}$ & 1000 & 45 & 59 & 4 & 0.1 & .26 & 2 & -.028 \\
\hline 301 & $1 \times 10^{-4}$ & $5 \times 10^{-4}$ & 1000 & 45 & 59 & 2 & 0.1 & .28 & 2 & -.038 \\
\hline 302 & $1 \times 10^{-4}$ & $5 \times 10^{-4}$ & 1000 & 45 & 59 & 1 & 0.1 & .07 & 1 & -.006 \\
\hline 303 & $1 \times 10^{-4}$ & $5 \times 10^{-4}$ & 1000 & 45 & 59 & 6 & 0.1 & .22 & 3 & -.047 \\
\hline 304 & $1 \times 10^{-4}$ & $5 \times 10^{-4}$ & 1000 & 45 & 59 & 8 & 0.1 & .21 & 3 & -.040 \\
\hline 305 & $1 \times 10^{-4}$ & $5 \times 10^{-4}$ & 1000 & 45 & 59 & 10 & 0.1 & .21 & 5 & -.048 \\
\hline 306 & $1 \times 10^{-4}$ & $5 \times 10^{-4}$ & 1000 & 45 & 59 & 0.75 & 0.1 & .02 & 1 & -.0008 \\
\hline 307 & $1 \times 10^{-4}$ & $5 \times 10^{-4}$ & 1000 & 45 & 59 & 0.50 & 0.1 & -.01 & 1 & -.0002 \\
\hline 308 & $1 \times 10^{-4}$ & $5 \times 10^{-4}$ & 1000 & 45 & 59 & 0.2 & 0.1 & -.08 & 1 & $-4 \times 10^{-}$ \\
\hline 310 & $1 \times 10^{-4}$ & $5 \times 10^{-4}$ & 1000 & 45 & 0 & 4 & 0.1 & .70 & 1 & $-2 \times 10^{-}$ \\
\hline 315 & $1 \times 10^{-4}$ & $5 \times 10^{-4}$ & 1000 & 45 & 59 & 4 & 0.005 & .10 & 2 & -.0001 \\
\hline 316 & $1 \times 10^{-4}$ & $5 \times 10^{-4}$ & 1000 & 45 & 59 & 4 & 0.025 & .21 & 2 & -.002 \\
\hline 317 & $1 \times 10^{-4}$ & $5 \times 10^{-4}$ & 1000 & 45 & 59 & 4 & 0.25 & .28 & 4 & -.118 \\
\hline 318 & $1 \times 10^{-4}$ & $5 \times 10^{-4}$ & 1000 & 45 & 59 & 4 & 10.0 & .29 & $\mathrm{C}$ & -.390 \\
\hline 325 & $1 \times 10^{-4}$ & $1 \times 10^{-4}$ & 1000 & 45 & 59 & 4 & 0.1 & .34 & $\mathrm{C}$ & -.113 \\
\hline 326 & $1 \times 10^{-4}$ & $2.5 \times 10^{-4}$ & 1000 & 45 & 59 & 4 & 0.1 & .29 & 3 & -.057 \\
\hline 327 & $1 \times 10^{-4}$ & $7.5 \times 10^{-4}$ & 1000 & 45 & 59 & 4 & 0.1 & .25 & 2 & -.017 \\
\hline 328 & $1 \times 10^{-4}$ & $10 \times 10^{-4}$ & 1000 & 45 & 59 & 4 & 0.1 & .24 & 2 & -.011 \\
\hline 329 & $1 \times 10^{-4}$ & $0.5 \times 10^{-4}$ & 1000 & 45 & 59 & 4 & 0.1 & .13 & $\mathrm{C}$ & -.061 \\
\hline 330 & $0.1 \times 10^{-4}$ & $5 \times 10^{-4}$ & 1000 & 45 & 59 & 4 & 0.1 & .05 & 1 & -.017 \\
\hline 331 & $0.5 \times 10^{-4}$ & $5 \times 10^{-4}$ & 1000 & 45 & 59 & 4 & 0.1 & .27 & 2 & -.039 \\
\hline 332 & $1.2 \times 10^{-4}$ & $5 \times 10^{-4}$ & 1000 & 45 & 59 & 4 & 0.1 & .29 & 3 & -.044 \\
\hline 335 & $1 \times 10^{-4}$ & $5 \times 10^{-4}$ & 1000 & 90 & 59 & 4 & 0.1 & .27 & 2 & -.019 \\
\hline 336 & $1 \times 10^{-4}$ & $5 \times 10^{-4}$ & 1000 & 30 & 59 & 4 & 0.1 & .23 & 3 & -.035 \\
\hline 337 & $1 \times 10^{-4}$ & $5 \times 10^{-4}$ & 1000 & 18 & 59 & 4 & 0.1 & .25 & 3 & -.062 \\
\hline $338 \mathrm{~F}$ & $1 \times 10^{-4}$ & $5 \times 10^{-4}$ & 1000 & 15 & 59 & 4 & 0.1 & .25 & 2 & -.016 \\
\hline $339 \mathrm{~F}$ & $1 \times 10^{-4}$ & $5 \times 10^{-4}$ & 1000 & 10 & 59 & 4 & 0.1 & .33 & 2 & -.022 \\
\hline 340 & $1 \times 10^{-4}$ & $5 \times 10^{-4}$ & 1000 & 45 & 20 & 4 & 0.1 & .18 & 2 & -.018 \\
\hline 341 & $1 \times 10^{-4}$ & $5 \times 10^{-4}$ & 1000 & 45 & 40 & 4 & 0.1 & .27 & 2 & -.033 \\
\hline 342 & $1 \times 10^{-4}$ & $5 \times 10^{-4}$ & 1000 & 45 & 79 & 4 & 0.1 & .22 & 2 & -.028 \\
\hline 350 & $1 \times 10^{-4}$ & $5 \times 10^{-4}$ & 1000 & 45 & 59 & $8,4,2^{*}$ & - & .23 & - & -.046 \\
\hline $351 \dagger$ & $1 \times 10^{-4}$ & $5 \times 10^{-4}$ & 1000 & 45 & 59 & $4,0.6,0.4^{*}$ & - & .27 & - & -.029 \\
\hline $352 \dagger$ & $1 \times 10^{-4}$ & $1 \times 10^{-4}$ & 1000 & 45 & 59 & $4,0.6,0.4 *$ & - & .24 & - & -.011 \\
\hline $353 \dagger$ & $1 \times 10^{-4}$ & $5 \times 10^{-4}$ & 1000 & $90,45,30 *$ & 85 & $4,0.6,0.4 *$ & - & .24 & - & -.050 \\
\hline 354 & $1 \times 10^{-4}$ & $5 \times 10^{-4}$ & 1000 & $90,45,30^{*}$ & 85 & 4 & 0.1 & .24 & 2 & -.051 \\
\hline 360 & $1 \times 10^{-4}$ & $5 \times 10^{-4}$ & 1100 & 45 & 59 & 4 & 0.1 & .52 & 2 & -.045 \\
\hline 361 & $1 \times 10^{-4}$ & $5 \times 10^{-4}$ & 1500 & 45 & 59 & 4 & 1.0 & .64 & $\mathrm{C}$ & -.049 \\
\hline 363 & $1 \times 10^{-4}$ & $5 \times 10^{-4}$ & 2000 & 45 & 59 & 4 & 1.0 & .41 & 1 & -.007 \\
\hline 370 & $1 \times 10^{-4}$ & $2.5 \times 10^{-4}$ & 1000 & 30 & 59 & 4 & 0.1 & .30 & 3 & -.063 \\
\hline 371 & $1 \times 10^{-4}$ & $2.5 \times 10^{-4}$ & 1000 & 45 & 59 & 8 & 0.1 & .18 & 5 & -.090 \\
\hline 372 & $0.5 \times 10^{-4}$ & $1 \times 10^{-4}$ & 1000 & 45 & 59 & 4 & 0.1 & .41 & $\mathrm{C}$ & -.168 \\
\hline $373 \mathrm{~F}$ & $1 \times 10^{-4}$ & $5 \times 10^{-4}$ & 1000 & 10 & 59 & 8 & 0.1 & .30 & 3 & -.024 \\
\hline $374 \mathrm{~F}$ & $1 \times 10^{-4}$ & $5 \times 10^{-4}$ & 1000 & 10 & 59 & 12 & 0.1 & .29 & 5 & -.024 \\
\hline $375 \mathrm{~F}$ & $1 \times 10^{-4}$ & $5 \times 10^{-4}$ & 1000 & 10 & 59 & 8 & 0.1 & .61 & $\mathrm{C}$ & -.099 \\
\hline $380 \div$ & $1 \times 10^{-4}$ & $5 \times 10^{-4}$ & 1000 & - & 0 & 4 & 0.1 & .17 & 1 & -.002 \\
\hline $381 \ddagger$ & $1 \times 10^{-4}$ & $5 \times 10^{-4}$ & 1000 & - & 0 & 4 & 0.5 & .29 & 1 & -.024 \\
\hline 385 & $1 \times 10^{-4}$ & $2.5 \times 10^{-4}$ & 500 & 45 & 30 & 4 & 0.1 & .27 & 4 & -.091 \\
\hline 386 & $1 \times 10^{-4}$ & $2.5 \times 10^{-4}$ & 333 & 45 & 20 & 4 & 0.1 & .26 & 2 & -.135 \\
\hline 390 & $1 \times 10^{-4}$ & $5 \times 10^{-4}$ & 1100 & 45 & 148 & 4 & 0.1 & .24 & 2 & -.042 \\
\hline $395 \mathrm{~s}$ & $1 \times 10^{-4}$ & $5 \times 10^{-4}$ & 1000 & 45 & 59 & 4 & 0.1 & .23 & 2 & -.031 \\
\hline $396 \mathrm{~S}$ & $1 \times 10^{-4}$ & $5 \times 10^{-4}$ & 1000 & 45 & 59 & 4 & 0.1 & .33 & 2 & -.032 \\
\hline
\end{tabular}

*Multiple forcing periods or wavelengths, with differing amplitudes for each component.

$\dagger$ Runs excluded from model comparisons because of mixed frequencies.

\$Wind stress varies alongshore with wavelength $45 \mathrm{~km}$.

Mult: C: Velocity time series are chaotic; 3: frequency tripling; 2: frequency doubling; 1: no frequency multiplication.

s: topography stretched in $x$ by $25 \%$; S: topography squeezed in $x$ by $25 \%$; F: fine grid resolution in $y$. 
Table 2. Extensive, randomized topography model runs.

$\begin{array}{cccccc}\begin{array}{c}\text { Run } \\ \text { number }\end{array} & \begin{array}{c}d_{1} \\ \left(\mathrm{~N} / \mathrm{m}^{2}\right)\end{array} & \begin{array}{c}T \\ \text { (days) }\end{array} & \begin{array}{c}\Lambda^{x} \\ (\mathrm{~km})\end{array} & \begin{array}{c}\Lambda^{y} \\ (\mathrm{~km})\end{array} & \Xi \\ 101 & 0.25 & 4 & 6.1 & 7.3 & 0.71 \\ 102 & 0.25 & 4 & 12.9 & 9.3 & 0.67 \\ 103 & 0.25 & 4 & 9.6 & 13.1 & 0.72 \\ 104 & 0.125 & 4 & 9.6 & 13.1 & 0.67\end{array}$

where the sum is generally truncated at one term, but sometimes up to three terms are used (with amplitudes decreasing with increasing frequency). The wind stress properties are summarized in Table 1 as amplitude $d_{1}$ and period $\left(T=2 \pi / \omega_{1}\right)$ for each run.

The model runs described in Sections 3 and 4 all use a two-slope topography (with background slopes $A$ and $B$ ) with alongshore corrugations super-imposed (Fig. 1). These runs are meant to be representative of continental shelf-slope topography. Specifically, the topography is described by

$$
\begin{array}{ll}
h(x, y)=h_{A}+h_{0}+A x+h_{1} G(y) x / x_{1} & \text { for } \quad 0<x<x_{1} \\
h(x, y)=h_{A}+h_{1}+B\left(x-x_{1}\right)+h_{1} G(y)\left(x_{2}-x\right) /\left(x_{2}-x_{1}\right) & \text { for } \quad x_{1}<x<x_{2} \\
h(x, y)=h_{A}+h_{1}+B\left(x_{2}-x_{1}\right) \equiv h_{A}+h_{2} & \text { for } \quad x_{2}<x
\end{array}
$$

where $h_{1}=h_{0}+A x_{1}$ and $h_{A}$ is a constant. In most cases, $x_{1}=40 \mathrm{~km}, x_{2}=60 \mathrm{~km}, h_{0}=$ $25 \mathrm{~m}, h_{1}=100 \mathrm{~m}$, and $h_{2}=1000 \mathrm{~m}$. For runs 360, 361, 363 and $390, h_{A}=100 \mathrm{~m}$, $500 \mathrm{~m}, 1000 \mathrm{~m}$ and $100 \mathrm{~m}$ respectively, but otherwise $h_{A}=0$. In Table 1, the maximum

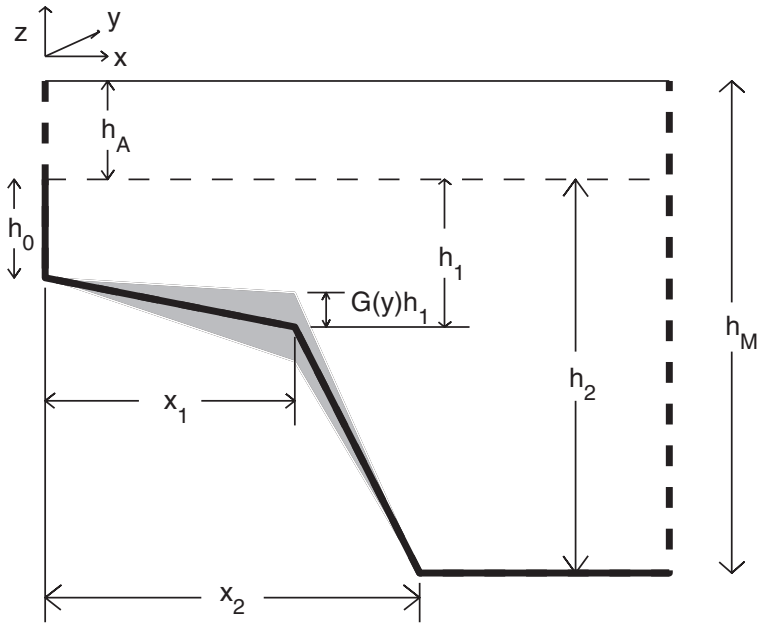

Figure 1. Definition sketch for the geometry (Eqs. 3). The shaded area denotes the form and amplitude over which alongshore depth variations occur. 
depth $h_{M}$ is provided as a way to characterize the overall depth. The alongshore topographic variations are described by

$$
G(y)=\sum_{n} \gamma_{n} \sin \left(k_{n} y\right)
$$

where the sum is often truncated at one term. For topography with more than one sinusoidal component (runs 353 and 354), the amplitudes are $\left(\gamma_{1}, \gamma_{2}, \gamma_{3}\right)=(0.1,0.3,0.55)$ for wavelengths of $(90,45,30) \mathrm{km}$. Table 1 characterizes the corrugations by means of $h_{C}$, the amplitude of the alongshore depth variations at the shelf break (i.e., at their offshore maximum location), and of $\lambda$, the alongshore wavelength(s) of the variations.

In Section 5, an extensive randomized topography (with no spatially-averaged bottom slope) is used. For each run, the wind stress is spatially uniform and has a single frequency. The domain is again cyclic in $y$, closed at $x=0$ and open at large $x$. In this case, the depth is given by

$$
h(x, y)=h_{A}+C \sum_{n} \sum_{m} n^{-\mu} m^{-v} \sin \left(2 \pi n x / W+\varphi_{n}\right) \sin \left(2 \pi m y / W+\psi_{m}\right)
$$

where the sums each go from 1 to $55, W=328 \mathrm{~km}$, and the exponents are varied in order to achieve different correlation length scales (i.e., the integral of the autocorrelation function of $h$ from 0 to large lag). The phases $\varphi_{n}$ and $\psi_{n}$ are random numbers in the range of 0 to $2 \pi$. In all cases presented here, there are enough sinusoidal components that the correlation function tends toward zero for lags that are large ( $>50 \mathrm{~km}$ in practice), but still less than half of the domain size. The large-domain runs are summarized in Table 2 in terms of the correlation length scales $\Lambda^{x}$ and $\Lambda^{y}$, and of forcing amplitude. All large domain runs use $f=1 \times 10^{-4} 1 / \mathrm{sec}, h_{A}=500 \mathrm{~m}, r=5 \times 10^{-4} \mathrm{~m} / \mathrm{sec}$ and the standard deviation of depth is $43 \mathrm{~m}$.

A set of nondimensional numbers can be defined for this system, starting with a scale for velocity, $V=T_{W} /\left(\rho f h_{M}\right)$, where $T_{W}$ is a representative wind stress amplitude. Using this, a Rossby number can be defined as

$$
R=T_{W} /\left(\rho f^{2} W h_{M}\right)
$$

where $W=x_{2}$ is the distance from the coast to the flat deep ocean. Other parameters include a nondimensional frequency, wavenumber and friction parameter

$$
\begin{aligned}
& \sigma=\omega / f, \\
& k=2 \pi W / \lambda,
\end{aligned}
$$

and

$$
E=r /\left(f h_{M}\right),
$$


where $\lambda$ is the longest alongshore wavelength of the topography. The depth variations are characterized by

$$
\Theta=\left(h_{M}-h_{m}\right) / h_{M}
$$

and

$$
\Gamma=\chi / h_{M}
$$

where $h_{m}$ is the water depth at the coast and $\chi$ is the amplitude of alongshore depth variations at the shelfbreak. Finally, the free surface divergence is characterized by

$$
D=\left(f^{2} W^{2}\right) / g h_{M},
$$

which is always small for the model runs presented here. These numbers are used to guide the choice of numerical model run parameters, and to assure that no redundant calculations occur.

\section{Overview of shelf-slope runs}

One initial question is whether any artifacts are introduced by using a strictly cyclic model geometry. Specifically, would noncyclic conditions allow for a spatially averaged alongshore pressure gradient that might counteract the rectification found, for example, by HB? Appendix A shows that although an alongshore sea-surface tilt can form in a noncyclic setting, it does not substantially affect the alongshore mean flow in the central corrugated region. The main role of the alongshore gradient is to provide a partial geostrophic balance for any net cross-shelf flow required by alongshore variations in alongshore transport.

The next step, then, is to concentrate on the two-slope problem where the geometry is perfectly cyclic in the alongshore direction (i.e., there are no extended alongshore-uniform sections as there are in the Appendix A examples). Results are expressed as time averages over the last full forcing cycle of a given run. Run 300 (Fig. 2, Table 1) is a representative example. It uses topography with a single alongshore wavelength of $\lambda=45 \mathrm{~km}$, and is forced at a 4-day period. The pressure and downwave-tending mean flow fields roughly parallel the isobaths, but a closer inspection shows that the pressure field has a "sawtooth" shape such that the alongshore $(y)$ pressure gradient is stronger on the downstream side of a depression than on the upstream (greater $y$ ) side. Thus, the mean flow is stronger on the upstream side of a ridge than the downstream. Further, there is a tendency for lower pressure on the upstream side of a ridge than on the downstream. Overall, the strongest mean flow (downwave at $0.028 \mathrm{~m} / \mathrm{sec}$ ) is at the shelf break (near $x=40 \mathrm{~km}$ ), and it is found in conjunction with bottom elevations (ridges), but not at depressions.

Although the mean flow broadly follows isobaths in the downwave sense, the tendency is far from uniform. Both nearshore along ridges (e.g., near $y=30 \mathrm{~km}$ ), and over the outer shelf over depressions (e.g., near $y=50 \mathrm{~km}$ ), this example has closed pressure contours 


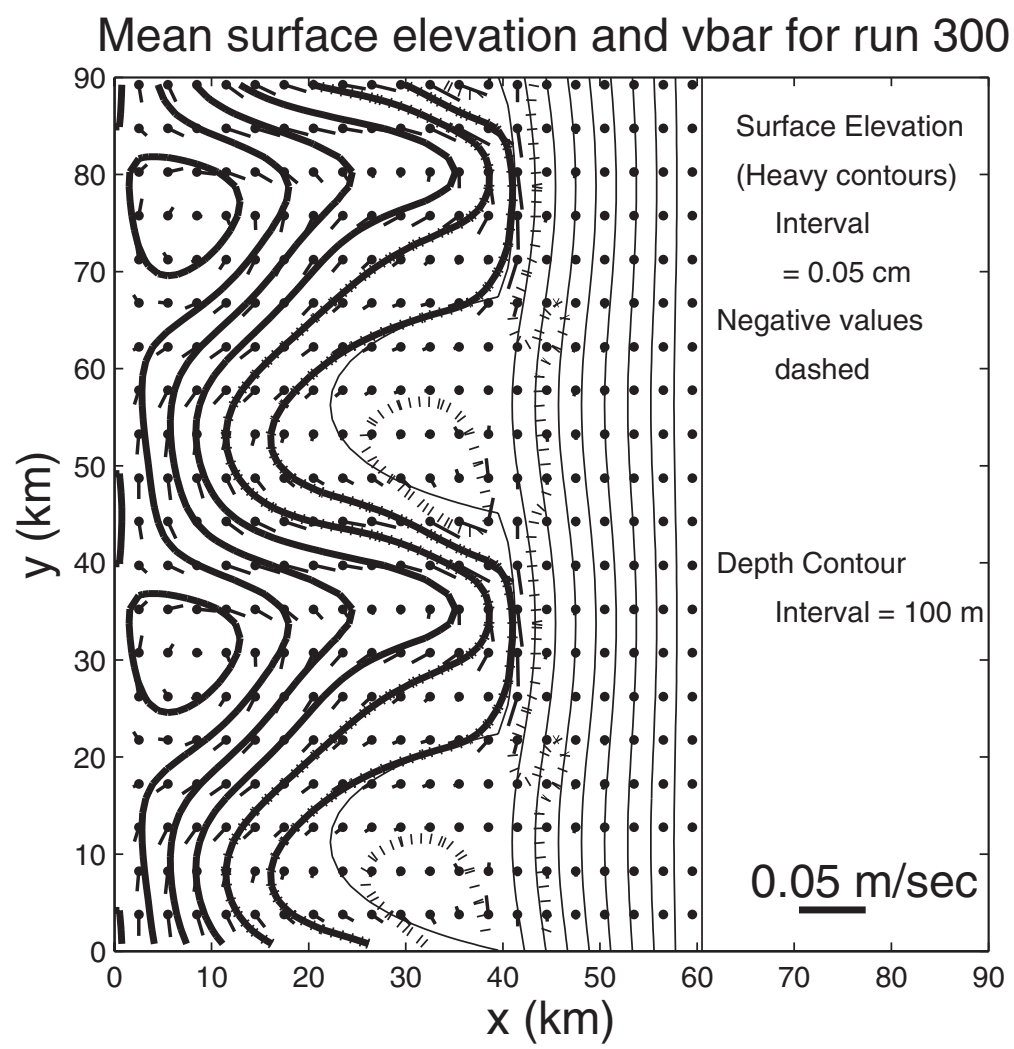

Figure 2. Mean (over the last forcing period) free surface height (m) for run 300, with solid heavy contours for positive and zero values, and heavy dashed contours for negative values. Depth contours are lighter solid lines (contour interval of $100 \mathrm{~m}$ ). Mean velocity vectors are also shown as short, light solid lines. The heavy dot is at the origin of the vector, i.e., the flow is away from the dot.

and closed, or nearly closed, circulations that cross isobaths. One way to quantify the net tendency for downwave flow along isobaths is to use the normalized topostrophy (Holloway, 2008):

$$
\Xi=\{f(\boldsymbol{k} \times \boldsymbol{v}) \cdot \nabla h\}\left[\left\{|f v|^{2}\right\}\left\{|\nabla h|^{2}\right\}\right]^{-1 / 2}
$$

where $\boldsymbol{v}$ is the vector depth-averaged velocity, $\boldsymbol{k}$ is the vertical unit vector, $\nabla h$ is the gradient of water depth, and \{ \} represents the mean of a quantity over a forcing period and over the entire spatial domain, except in deep water where the bottom is flat. The advantage of using topostrophy is that it is weighted so that weak flows do not make a major contribution. If the mean flow follows isobaths exactly, and all flow is downwave, then $\Xi$ is generally relatively large (but $\leq 1$ ). If the flow has no preferred orientation relative to isobaths, then $\Xi=0$. For run $300, \Xi=0.26$, a representative value for the two-slope runs 

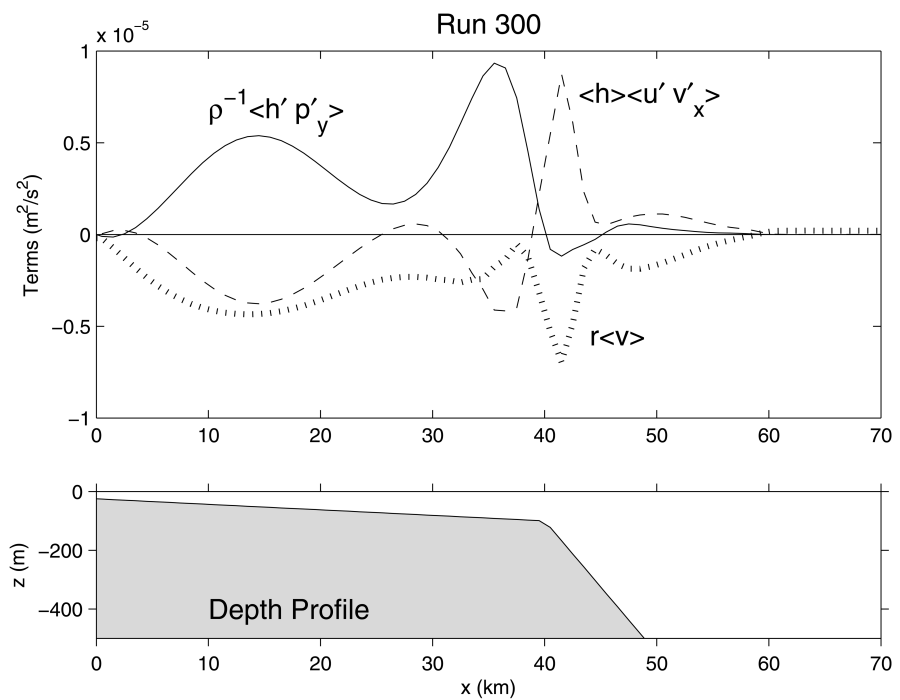

Figure 3. Upper panel: alongshore-averaged, time mean momentum balance terms for run 300 . The solid line is form stress, $\rho^{-1}\left\langle h^{\prime} p_{y}^{\prime}\right\rangle$, the dashed line is the cross-shelf momentum transport, $\langle h\rangle\left\langle u^{\prime} v_{x}^{\prime}\right\rangle$, and the dotted line is the bottom stress, $r\langle v\rangle$. Lower panel: alongshore-averaged depth profile. Only the upper $500 \mathrm{~m}$ is shown.

(Table 1, with most having $0<\Xi<0.5$ ). Thus, there is a distinct tendency for model mean flow to follow isobaths, but the correspondence is far from exact.

Is it reasonable to interpret the mean flow pattern in Figure 2 as being caused by topographic rectification? A straightforward way to answer this is to consider the depth-integrated alongshore momentum equation, averaged over time and in $y$ over an alongshore cycle, i.e.,

$$
\langle h\rangle\langle u\rangle\langle v\rangle_{x}+\cdots+\langle h\rangle\left\langle u^{\prime} v_{x}^{\prime}\right\rangle=-\rho^{-1}\left\langle h^{\prime} p_{y}^{\prime}\right\rangle-r\langle v\rangle,
$$

where a "prime" represents a deviation from the time-alongshore, \langle\rangle , mean. The ellipsis in (8) denotes generally small advective terms involving correlations of perturbation depth with perturbation velocities. Comparing the many runs, various subsets of the terms in (8) are important under some conditions, but the three terms that are consistently important are $\rho^{-1}\left\langle h^{\prime} p_{y}^{\prime}\right\rangle,\langle h\rangle\left\langle u^{\prime} v_{x}^{\prime}\right\rangle$ and $r\langle v\rangle$. These three terms are plotted as a function of cross-shelf distance in Figure 3 for run 300. The topographic stress $\left\langle h^{\prime} p_{y}^{\prime}\right\rangle$ is generally well-balanced by the combination of frictional drag $\rho r\langle v\rangle$ and cross-shelf eddy transport, $\rho\langle h\rangle\left\langle u^{\prime} v_{x}^{\prime}\right\rangle$. Further, if (8) is integrated over a volume (e.g., by integrating the information in Figure 3 across $x$ ), the integrated $\langle h\rangle\left\langle u^{\prime} v_{x}^{\prime}\right\rangle$ term is small relative to the other two terms shown in Figure 3.

In general, integrated over volume, the main balance in (8) is between topographic stress $\left\langle h^{\prime} p_{y}^{\prime}\right\rangle$ and damping $\rho r\langle v\rangle$. One might then expect that the primary role of the eddy term 
$\langle h\rangle\left\langle u^{\prime} v_{x}^{\prime}\right\rangle$ is to move alongshore momentum cross-shelf from where it is generated by the topographic stress to where it is dissipated by bottom friction. In the deep water run 363 , as an extreme, the time- and alongshore-averaged topographic stress is largely balanced locally (at the same $x$ ) by bottom stress over the entire sloping-bottom domain. It thus appears that mean pressure differences across topography play a major role in the dynamics of the mean alongshore currents: a finding consistent with the "Neptune" concept (e.g., Holloway, 1992).

Eq. (8) can be integrated over all $x$ (assuming that there is no momentum lost through the offshore boundary) to yield

$$
0=-\frac{1}{\rho} \llbracket h^{\prime} p_{y}^{\prime} \rrbracket-r \llbracket v \rrbracket
$$

where 【_』 represents an integral over all space and over time. This expression highlights the importance of topographic irregularities in this problem. If the isobaths were straight, or if $h^{\prime}$ were in quadrature with $p_{y}^{\prime}$, then the averaged bottom stress $r \llbracket v \rrbracket=0$, i.e., the averaged bottom stress would have to be zero. The quadrature relation would apply if the geostrophic velocity follows isobaths exactly, so flow exactly along isobaths is only to be expected in a system where $r=0$. On the other hand, for straight isobaths, the $r \llbracket v \rrbracket=0$ condition could place a very strong constraint on the final flow field.

The conclusion (Eq. 9) that, overall, the topographic stress balances bottom friction is reached by volume averaging in the $(x, y)$ coordinate system: a traditional, but not unique, way to view such a problem. One could imagine that, viewed locally and perhaps in some other coordinate system (such as following isobaths as in Section 4), the balances could look rather different.

Inspection of the model runs leads to some preliminary results about mean flow generation. For example, the mean downwave along-isobath velocity is always patterncorrelated with the standard deviation of cross-isobath flow (e.g., 0.31 for run 300, using 3660 model points), but not with the standard deviation of along-isobath flow (e.g., 0.02 for run 300 ). For context, the spatially averaged standard deviation of cross-isobath velocity is usually less than, or comparable to, that of along-isobath velocity (the respective values are 0.02 and $0.035 \mathrm{~m} / \mathrm{sec}$ for run 300). The mean along-isobath flow also tends to be strongest where $|\nabla h| / h$ is largest; e.g., near the shelfbreak in Figure 2. Thus, multiplying the cross-isobath standard deviation by $|\nabla h| / h$ enhances the pattern correlation with mean along-isobath flow. Merryfield et al. (2001) use their inviscid, initial-value eddy evolution model to predict a similar depth dependence, and thus also find that the strongest mean flow usually appears near the shelf break. Finally, if the mean flow is normalized by the standard deviation of cross-isobath velocity (which itself depends on $r, \omega$ and $h$, among other things) and many runs are compared, the frequency and frictional coefficients are only weakly correlated with mean flow for subinertial frequencies. Further, this normalized mean flow is weaker for higher forcing frequencies, for example in run 302, where the maximum mean along-isobath flow is $<0.01 \mathrm{~m} / \mathrm{sec}$ (Table 1 ). 


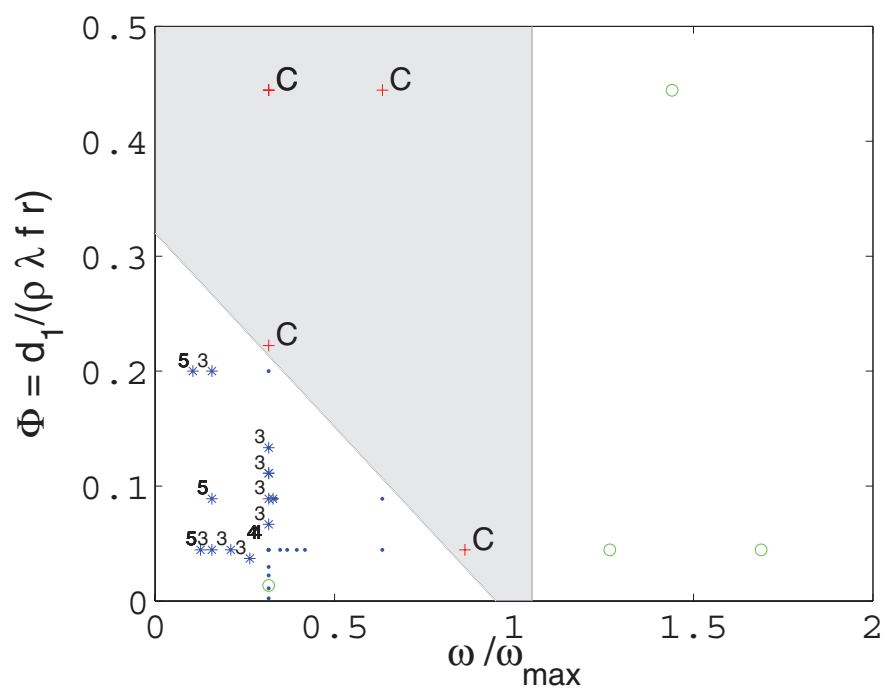

Figure 4. Regime diagram for frequency multiplication and chaos. Runs with no frequency multiplication are shown as green circles. Runs with frequency doubling are denoted by blue dots. Runs with higher frequency multiplication are shown as a blue asterisk along with a number denoting the degree of frequency multiplication. Chaotic runs are denoted by red crosses with the letter "C." Shading is used only to guide the eye to the area where chaotic runs are found. The edges are not meant to represent defined boundaries for chaos.

In most cases, the model does not simply respond at only the frequency of the forcing, but rather it shows temporally complex behavior reminiscent of that found in a simple ocean basin numerical model by Veronis (1963) for example. Samelson and Allen (1987) treated a very idealized, quasigeostrophic equivalent to the present problem, and found that for large forcing, weak damping or near-resonant (with topographic waves) conditions, the ocean's response can include frequency multiplication or chaotic behavior. Although the transition to chaos is not a central concern in the present study, a sampling of chaotic behavior is in fact obtained here. For the present purposes, "chaotic" is taken to mean that velocity time series somewhere in the domain are not obviously periodic, even though the forcing is strictly periodic and the start-up transients have died away. The chaotic runs here all have a broad frequency spectrum, including energy at frequencies higher than the forcing. Often, frequency multiplication and chaotic behavior are spatially confined to within a few kilometers of the shelf break. A regime diagram comparable to Samelson and Allen's Figure 6 can be created (Fig. 4), although their amplitude parameter is replaced with the ratio of the dissipative time scale $\left(h_{M} / r\right)$ to the time scale of alongshore advection over a wavelength $\rho \lambda f h_{M} / d_{1}$, i.e., with $\Phi=d_{1} /(\rho \lambda f r)$. Further, the horizontal coordinate $\left(\omega / \omega_{\max }\right)$ is the forcing frequency divided by magnitude of the maximum frequency for a gravest mode shelf wave dispersion curve. The maximum frequency is crudely estimated, following Gill (1982), as $\omega_{\max }=f_{s} /\left(l^{2}+s^{2}\right)^{1 / 2}$, where $l=\pi / x_{2}, s \approx 0.5 \alpha / H, H$ is the 
mean depth for $x<x_{2}$, and $\alpha$ is the mean bottom slope. This sorting of the model results separates chaotic model runs (all within the shaded area of Fig. 4) cleanly from the others. No chaos or frequency multiplication is found for $\left(\omega / \omega_{\max }\right)>1$, i.e., for frequencies higher than the maximum for a shelf wave. Further, there are no chaotic runs found for $\Phi<0.32\left[1-\left(\omega / \omega_{\max }\right)\right]$. Needless to say that the parameter space is not thoroughly sampled, so that the regime boundaries are not actually as well-defined as the shading in Figure 4 might suggest. Another interesting feature in this diagram is the "island" of higher frequency multiplication for smaller $\left(\omega / \omega_{\max }\right)$ and $\Phi$. The reason for this island is not obvious, but it should be noted that other natural frequency scales (associated with higher mode coastal-trapped waves) ought to enter the problem and so complicate the regime diagram.

\section{Mean flow parameterization}

The strength of the mean flow ought to be expressible in terms of other observable model quantities. For example, preliminary inspection (Section 3) of the model outputs is encouraging in that the mean flow is distinctly correlated with bottom slope and crossisobath flow variability. These correlations suggest that the most appropriate coordinate system is one that follows isobaths exactly. Thus an attempt is made here to find expressions, based on physical reasoning, that encapsulate the numerical model mean flow results.

Consider a coordinate system where $\eta$ is perpendicular to the local isobaths (positive toward deeper water), and $\zeta$ is along isobaths (positive upwave). Thus, $h_{\zeta} \equiv 0$ and

$$
\begin{aligned}
u_{t}^{\dagger}+N^{\eta}-f v^{\dagger} & =-\rho^{-1} p_{\eta}-r u^{\dagger} / h+\rho^{-1} \tau^{\eta} / h \\
v_{t}^{\dagger}+N^{\zeta}+f u^{\dagger} & =-\rho^{-1} p_{\zeta}-r v^{\dagger} / h+\rho^{-1} \tau^{\zeta} / h, \\
0 & =\nabla \cdot(h v)
\end{aligned}
$$

where $u^{\dagger}$ and $v^{\dagger}$ are the velocity components in the ( $\left.\eta, \zeta\right)$ directions, respectively, and $N^{\eta}$ and $N^{\zeta}$ are the advective acceleration terms in the along-isobath coordinate system.

The scaling first builds on analogy to Huthnance (1973) or Loder (1980), where the flow field is thought of as having a zero-mean forcing that gives rise to a linear, oscillating flow at lowest order (subscript "0"). At the next order (subscript "1"), system (10) yields equations for the mean flow velocity, where the steady forcing arises from the nonlinear terms $N^{\eta}$ and $N^{\zeta}$. Conceptually, this approach can be rationalized either by perturbation in a Rossby number, or by spectral truncation. The time average of (10b) over a forcing period is then

$$
\overline{N_{0}^{\zeta}}+\overline{f u_{1}^{\dagger}}=-\rho^{-1} \overline{p_{1 \zeta}}-r \overline{v_{1}^{\dagger}} / h
$$

where $\overline{N_{0}^{\zeta}}$ is the along-isobath advective acceleration estimated from the lowest order (zero mean) response and an overbar denotes a time mean. If this equation is averaged 
along-isobath over the cyclic channel length (which is an integral number of topographic wavelengths), the net cross-isobath flow is zero, and (11) reduces to

$$
\left\{\overline{N_{0}^{\zeta}}\right\}_{C}=-r\left\{\overline{v_{1}^{\ddagger}}\right\}_{C} / h,
$$

where \{\}$_{C}$ is an along-isobath average over a cycle of the repeating topography. Relation (12) is evaluated using the model runs in Table 1 (minus those with multiple forcing frequencies, wavelength $90 \mathrm{~km}$ and the closed-isobath case, run 390) by estimating the nonlinear terms in Cartesian coordinates and then rotating into local along-isobath coordinates. The result is that the correlation of the two terms in (12) is 0.93 and their regression coefficient is 1.2 (correlation of 0.99 and regression 0.97 if chaotic runs, where a steady state is not reached, are also dropped), evidence for the underlying balance of dissipative and eddy momentum transport effects for along-isobath flow. Of course, this high level of agreement is not surprising given that, by averaging over an along-isobath cycle, the only other surviving term besides those in (12) would be the time derivative. Note how strikingly this balance differs from the topographic stress balance obtained in Section 3. The difference occurs because the along-isobath topographic stress term is zero everywhere in the $(\eta, \zeta)$ system.

Physically, the forcing term $\overline{N_{0}^{\zeta}}$ in (11) represents the accumulation of along-isobath momentum due to cross-isobath fluctuating flow (e.g., Huthnance, 1973). The term ought to be mathematically more complicated here than in the straight-isobath case, but it might still be expected to increase (1) with increasing bottom slope, and (2) as stronger frictional effects break down the temporal quadrature phase relationship between the orthogonal velocity components.

The mathematics can be considerably simplified by making the "long wave" approximation (e.g., Gill and Schumann, 1974) for the steady flow component, i.e. by assuming that frictional effects are not too strong: $E^{2} \ll 1$ and that along-isobath scales $L^{\zeta}$ are greater than cross-isobath scales $L^{\eta}$. Included in this assumption is the idea that curvature and isobath-divergence effects are not too severe. The length scale assumption is not generally valid in the present problem, but the assumption does help clarify the physics. Under these conditions, the mean along-isobath flow is geostrophically balanced

$$
-\overline{f v_{1}^{\dagger}}=-\rho^{-1} \overline{p_{1 \eta}},
$$

and a governing equation for the time-mean pressure field (hence flow field) is found using (11), (13) and (10c):

$$
0=r \overline{p_{1 \eta \eta}}+f h_{\eta} \overline{p_{1 \zeta}}+\rho f\left(h \overline{N_{0}^{\zeta \zeta}}\right)_{\eta} .
$$

This parabolic equation, treated, for example by Csanady (1978) in a wind-driven context, shows that the ocean's mean-flow response to the eddy forcing $\overline{N_{0}^{\zeta}}$ is displaced downwave along isobaths; i.e., in the sense of free long, coastal-trapped wave propagation: no information can spread upwave. Csanady explained the useful analogy of this equation to 
the heat equation, where $-\zeta$ is analogous to time and $\left[r /\left(f h_{\eta}\right)\right]$ to a diffusivity. The exact amount of spatial offset between forcing and response depends upon the parameters $r, f$ and $h_{\eta}$. If the long-wave approximation were not made, (14) would be replaced by an elliptical equation that would allow some information to spread upwave, but would still, for reasonable parameter choices, preferentially spread the response downwave. (This is analogous to eastward and westward Rossby wave radiation, respectively, on a $\beta$ plane.) It is important to note that the spatial offset between the forcing and the pressure response allows for an overall correlation (integrated over $x$ and $y$ ) of bottom slope and pressure. That is to say that the isobaths and pressure contours are not expected to be exactly parallel. If isobars and isobaths were always parallel (i.e., $h$ and $p$ overlie each other exactly), $h^{\prime}$ and $p_{y}^{\prime}$ would always be in spatial quadrature, and no net topographic stress would occur in Eq. (9). The physics of how the pressure field sets up relative to the topography is discussed for an analogous quasigeostrophic model by Holloway (1987).

A simple order-of-magnitude estimate for the mean flow response arises from conjecturing that along-isobath pressure variations in (14) are negligible (or, equivalently, that the time-mean bottom Ekman transport dominates over cross-isobath geostrophic flow), so that

$$
0 \approx r \overline{p_{1 \eta \eta}}+\rho\left(h \overline{N_{0}^{\zeta}}\right)_{\eta}
$$

or (from Eq. 11)

$$
\overline{v_{1}^{\dagger}} \approx-h \overline{N_{0}^{\zeta}} / r
$$

locally, in contrast to (12) which holds in an averaged sense. This idealization implies that the mean flow is strictly along isobaths ( $\Xi$ approaches 1 ), so isobars parallel isobaths and the topographic stress $\left\langle h^{\prime} p_{y}^{\prime}\right\rangle=0$ everywhere. Model runs always have $\Xi<1$, and so it is obvious that (15b) is at best an approximation. Expression (15b) is analogous to e.g., Loder's (1980) balance for tidal rectification, where he assumed from the outset that there are no velocity variations along his straight isobaths. For the present problem, (15b) is taken to represent only a typical magnitude of the mean flow.

Parameterization of a typical mean flow now requires estimating the cross-isobath eddy momentum transport, preferably in a way that makes use of quantities readily observed in the ocean. One clue is that the numerical experiments show that the mean along-isobath flow is correlated with the standard deviation of cross-isobath velocity, but not with that of along-isobath flow. Further, the model along-isobath eddy momentum transport $\overline{v_{0}^{\dagger} v_{0 \zeta}^{\dagger}}$ is found to be uncorrelated with mean along-isobath flow. Thus, it appears that the dominant contributions to $\overline{N_{0}^{\zeta}}$ are related to the cross-isobath flux of alongshore momentum, centripetal terms, and effects related to isobath divergence/convergence. If the isobaths are relatively straight and parallel (e.g., if cross-isobath scales of variation are shorter than the radius of curvature), $\overline{N_{0}^{\zeta}}$ must be dominated by the eddy flux divergence, $\overline{u_{0}^{\dagger} v_{0 \eta}^{\dagger}}$. This term is now estimated by conjecturing that 


$$
\overline{u_{0}^{\dagger} v_{0 \eta}^{\dagger}} \approx c S D\left(u_{0}^{\dagger}\right) S D\left(q_{0 \eta}^{\dagger}\right)
$$

where $S D()$ denotes the standard deviation of a quantity, $c$ is a constant, and $q_{0 \eta}^{\dagger}$ is the temporal component of $v_{0 \eta}^{\dagger}$ that is in phase with $u_{0}^{\dagger}$. The important point, motivated by the de-phasing role of friction in Huthnance (1973), for example, is to concentrate on only $v_{0 \eta}^{\dagger}$ variations that are in phase with $u_{0}^{\dagger}$.

An example helps to motivate (16), which is central to the scaling argument. With straight isobaths, and an imposed oscillating cross-isobath flow $[U / h(x)] \sin (\omega t)$, the cross-isobath gradient of the along-isobath fluctuating flow is

$$
v_{0 \eta}^{\dagger}=v_{0 x}=\frac{U f h_{x}}{\omega^{2} h^{2}}[(2 r / h) \sin (\omega t)-f \cos (\omega t)] .
$$

However, because only the $\sin (\omega t)$ component of (17) contributes to the time average $\overline{u_{0}^{\dagger} v_{0 \eta}^{\dagger}}$,

$$
q_{0 \eta}^{\dagger}=\frac{U f h_{x}}{\omega^{2} h^{2}}(2 r / h) \sin (\omega t)
$$

and

$$
\overline{u_{0}^{\dagger} v_{0 \eta}^{\dagger}}=\frac{r U^{2} f h_{x}}{\omega^{2} h^{4}} .
$$

Applying (15b) then yields

$$
\overline{v_{1}^{\dagger}}=\overline{v_{1}}=-\frac{U^{2} f h_{x}}{\omega^{2} h^{3}} .
$$

For future reference, if the cross-isobath flow is given on the actual isobath of interest: $U=\widehat{u_{0}} h_{0}$ and $h_{0} \approx h$, then

$$
\overline{v_{1}} \approx-\frac{\widehat{u}_{0}^{2} f h_{x}}{\omega^{2} h} .
$$

The $q_{0 \eta}^{\dagger}$ term in (16) can be estimated for the present problem, in the "long wave" limit, by using potential vorticity conservation for the time-varying lowest-order flow:

$$
Q=\left[f+v_{0 \eta}^{\dagger}\right] / h=f / h_{0}
$$

where $h_{0}$ is the initial depth of a water column, dissipative terms are neglected because $E^{2} \ll 1$, and the relative vorticity of the mean flow is assumed to be negligible. Thus, the relative vorticity obtained by a water parcel as it crosses isobaths is

$$
v_{0 \eta}^{\dagger}=f \Delta h / h_{0} \approx f \Delta \eta h_{\eta} / h_{0} .
$$


What then sets the displacement $\Delta \eta$ ? The temporal component of $\Delta \eta$ that contributes to $q_{0 \eta}^{\dagger}$, hence the eddy transport, is that associated with the cross-shelf Ekman velocity due to the along-isobath flow $v_{0}^{\dagger} r /(f h)$. Thus,

$$
\Delta \eta=\Delta t v_{0}^{\dagger} r /(f h)=L^{\zeta} r /(f h),
$$

where $L^{\zeta}=\Delta t v_{0}^{\dagger}$ is an along-isobath excursion over time $\Delta t$.

If the isobaths were perfectly straight, or nearly so, the appropriate excursion scale is simply the integral of the fluctuating along-isobath velocity:

$$
L^{\zeta} \approx \widehat{v_{0}^{\dagger}} / \omega \approx \widehat{u_{0}^{\dagger}} f / \omega^{2},
$$

where $\widehat{u_{0}^{\dagger}}$ and $\widehat{v_{0}^{\dagger}}$ are the amplitudes of the fluctuating velocity. Thus,

$$
\Delta \eta=\widehat{u_{0}^{\dagger}} r /\left(h \omega^{2}\right)
$$

and, using (16),

$$
\overline{u_{0}^{\dagger} v_{0 \eta}^{\dagger}} \approx \operatorname{bfrSD}\left(u_{0}^{\dagger}\right)^{2} h_{\eta} /\left(h^{2} \omega^{2}\right),
$$

where $b$ is an unknown constant and (using 15b),

$$
\overline{v_{1}^{\dagger}}=-a_{\omega} f h_{\eta} S D(u \perp)^{2} /\left(h \omega^{2}\right),
$$

where $S D(u \perp)$ is the standard deviation of velocity perpendicular to the isobath (used in place of the amplitude), and $a_{\omega}$ is a coefficient to be determined empirically from the numerical model runs (accounting for the relation between standard deviation and amplitude, $a_{\omega}=2$ for straight isobaths from Eq. 20b or from Loder (1980), Eq. 29 for small $h-h_{0}$ ). Thus, if the isobaths are locally straight by some measure, the scaling recovers the analytical result.

If the along-isobath displacement is comparable to topographic scales, oscillating water parcels encounter varying isobath curvature and divergence, as well as a different cross-isobath flow during their excursions. For example, if the lowest order flow is spatially uniform, a water parcel moves toward shallow water in one location but toward deeper water half a topographic wavelength away in the $y$ direction. Thus, for larger particle excursions, the appropriate along-isobath scale is the topographic scale $L^{T}$ (either a topographic wavelength, or a correlation scale when topography is genuinely irregular). Thus,

$$
\Delta \eta=b^{*} L^{T} r /(f h),
$$

where $b^{*}$ is an unknown constant, and using (22),

$$
q_{0 \eta}^{\dagger}=b^{*} r h_{\eta} L^{T} / h^{2} .
$$

(15b) and (16) then yield 


$$
\overline{v_{1}^{\dagger}} \approx-a_{T} S D(u \perp) L^{T} \operatorname{sign}(f) h_{\eta} / h,
$$

where $a_{T}$ is determined empirically, and the $\operatorname{sign}(f)$ assures that the mean flow is directed downwave. Consistent with (30) representing the case where $L^{T}$ is short relative to an along-isobath particle excursion, the weaker of the two velocities (27) or (30) will be more correct.

Up to this point, it has been assumed that the eddy momentum transport is associated with relatively weak flows and frictionally-induced phase offsets that allow the $u^{\dagger}$ and $v^{\dagger}$ component fluctuations to be out of quadrature and so transport momentum. As the fluctuating flow becomes stronger, the fluctuations become increasingly nonlinear, and it is possible that stirring alone is sufficient to transport moment, i.e., that it does not simply depend on frictionally induced phase shifts. Specifically, one can follow the line of argument often used for estimating eddy transports (e.g., Visbeck et al. (1996) for heat transport) and assume that

$$
\overline{u_{0}^{\dagger} v_{0 \eta}^{\dagger}}=a_{M} S D(u \perp) S D\left(v_{0 \eta}^{\dagger}\right)
$$

and, using (22) and $\Delta \eta \approx S D(u \perp) / \omega$,

$$
\overline{u_{0}^{\dagger} v_{0 \eta}^{\dagger}}=b^{\ddagger} f h_{\eta} S D(u \perp)^{2} /(\omega h),
$$

where $b^{\ddagger}$ is a correlation between $u \perp$ and $v_{0 \eta}^{\dagger}$. It is now straightforward to apply (15b) to obtain an expression for the strong-stirring limit

$$
v^{M}=-a_{M} f h_{\eta} S D(u \perp)^{2} /(r \omega),
$$

where the coefficient $a_{M}$ is to be found empirically. This frictionally bounded form should apply when it yields a weaker flow estimate than either of the two previous estimates (Eq. 27 or 30). Expression (33) was also derived, using a different approach, by Ou (1999), although Cummins (2000) criticizes Ou's assumptions.

The forms (27), (30), (33) for the mean velocity are only a gross approximation for the true local velocity, since (15b) is assumed. In reality, the eddy transports implicit in these expressions force a response (through Eq. 14 or its elliptical equivalent) that is spatially shifted somewhat downwave relative to the forcing. This systematic spatial offset (or, ultimately, failure for isobars to parallel isobaths) is critical for allowing the existence of a net topographic stress $\left\langle h^{\prime} p_{y}^{\prime}\right\rangle$ in the system.

The empirical coefficients in (27), (30) and (33) are evaluated using 39 of the runs in Table 1 (dropping three runs with mixed forcing frequencies, 3 with straight isobaths, and all 6 chaotic runs, which were never steady to within $25 \%$ from one forcing period to the next). The selection of runs deletes two (380 and 381) with smooth topography but winds 


\section{Mean along-isobath flow parameterization}

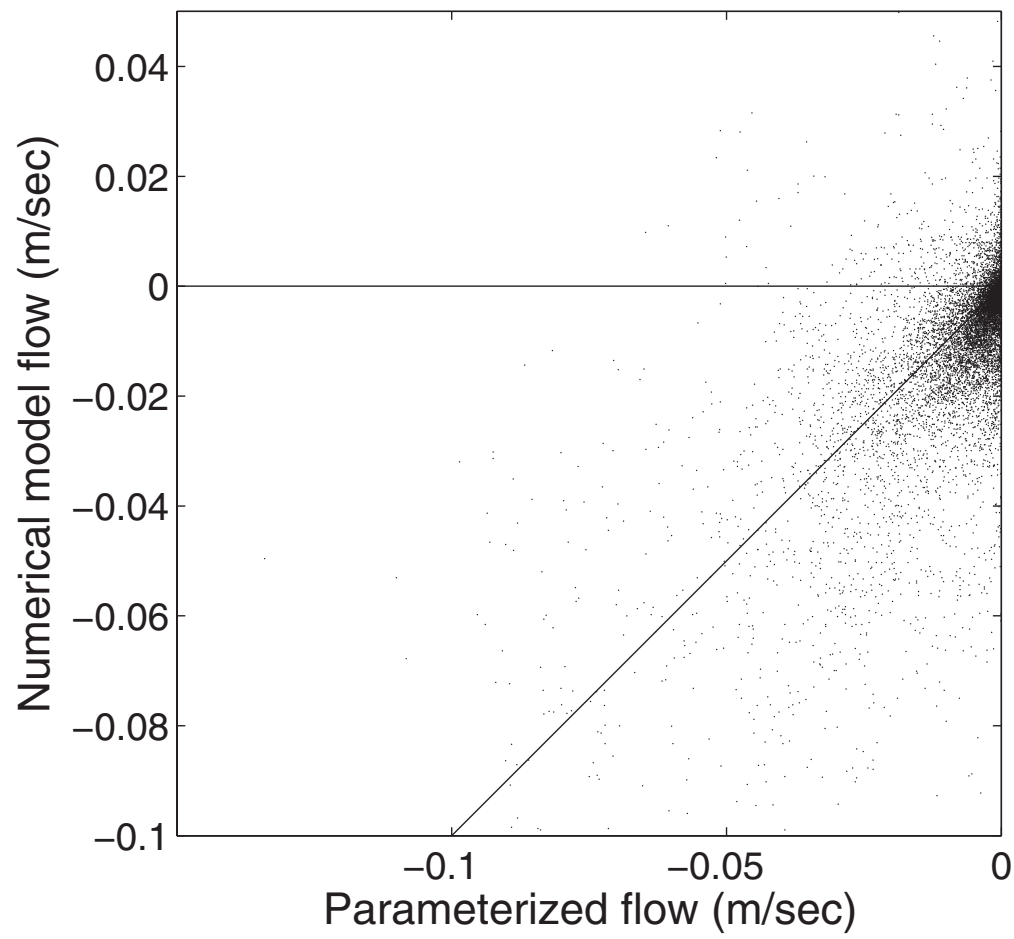

Figure 5. Scatter plot comparing model mean velocity parallel to isobaths to the parameterization using the weakest of (27), (30) and (33). The plot uses only one in 11 of the points that were used to evaluate coefficients.

that vary sinusoidally alongshore, because constraint (9) implies that a different parameterization will apply in this case. The above model (the minimum magnitude of 27, 30, and 33) for mean along-isobath flow is then compared to every point with a sloping bottom in all of the 39 runs. The coefficients $a_{T}, a_{\omega}$ and $a_{M}$ are then optimized in a least-squares sense over a total of 142,740 spatial points. This is a nonlinear least-squares problem because of the conditional nature of the solution, and so it is solved iteratively. It is likely that the present optimization is unique because some effort was spent seeking alternative optimizations, but none were found.

The overall fit $\left(a_{T}, a_{\omega}, a_{M}\right)=(0.23,0.55,0.23)$ accounts for $60 \%$ of the total energy in mean along-isobath velocity (Fig. 5). In this optimal fit, the topographically constrained form (30) applies at $10 \%$ of the points, the traditional "tidal rectification" form (27) applies at $61 \%$ of the points, and the homogenization estimate (33) applies at $29 \%$ of the points. If only the topographically constrained form (30) were used (and $a_{T}$ re-optimized), it would account for $50 \%$ of the along-isobath mean energy. If the along-isobath-uniform rectification form (27) were used exclusively (and $a_{\omega}$ re-optimized), it would account for $36 \%$ of 
the energy. Finally, if only the limiting speed (33) were used, it could account for $43 \%$ of the energy. The point is that for the present selection of runs (which is admittedly biased because it does not include any alongshore scales that are much greater than the shelf width, for example), the topographically constrained model (30) is the single best representation of the numerical model results.

The arguments leading to (27), (30) or (33) assume that the cross-isobath eddy

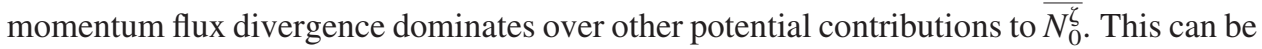
evaluated directly using a set of 42 runs. Using the parameterized eddy momentum flux divergences implicit in $(26,30$ and 32) in an evaluation of the along-isobath averaged balance (11) yields a correlation of $\left\{\overline{N_{0}^{\zeta}}\right\}_{C}$ with $r\left\{\overline{v_{1}^{\dagger}}\right\}_{C} / h$ of -0.93 , nearly as good as that obtained by calculating the nonlinear terms directly: -0.99 (this calculation uses estimates along the 50,100,150, 200, 300, 400, 500, 600, 700, 800 and $900 \mathrm{~m}$ isobaths). Thus, the eddy transport parameterization works well in an along-isobath averaged sense. Comparing the parameterized (Eq. 16) and calculated flux divergences, $\overline{N_{0}^{\zeta}}$, point-by-point leads to a substantially worse result: a correlation of 0.43 . Inspection of the parameterized and computed $\overline{N_{0}^{\zeta}}$ shows that, while the along-isobath means generally agree, the directly calculated terms have a good deal more short-scale variability than do the parameterized transports. The two estimates do agree well point-by-point in the cases where bottom frictional effects (measured by $r /(f h)$ ) are less small. That is, stronger friction eliminates the shorter spatial scales in the mean eddy flux divergence. It thus appears that, in terms of the local momentum flux divergence $\overline{N_{0}^{\zeta}}$, alongshore fluxes and effects associated with isobath curvature and divergence, as well as with general nonlinear scale coupling, must be important, although the better (than correlation 0.43) local performance of the $\bar{v}$ parameterization in (12) or in $(27,30,33)$ suggests that the important aspects of the eddy flux divergence are somehow estimated with some skill.

How is it that a spatially ragged forcing field gives rise to along-isobath velocities that are well-estimated by a much smoother parameterized field? The parabolic nature of (14), which presumably only exaggerates the along-isobath asymmetries of its more correct elliptical equivalent, requires that the pressure response reflects a weighted average of the forcing upwave from a given location. This property assures that the response is spatially smoother than the forcing, and that it is offset in space (downwave in this case) relative to the forcing. Thus, there is no requirement that the mean pressure (hence current) solution bear a strong resemblance to the forcing locally. On the other hand, the parameterized velocity estimate $(27,30,33)$ has empirical coefficients that are optimized with regard to the localized limit (15b), which does imply that the pressure field and estimated (Eq. 16) forcing are comparably smooth. Thus, the poor point-by-point comparison of computed vs. parameterized forcing fields is justifiable. One implication of the spatially rough (compared to the mean along-isobath flow) nature of the eddy forcing field is that, on a point-by-point basis, there may be little or no correlation between the local eddy forcing and the local mean velocity in nature. Thus, the nonlocal, smoothed nature of the mean 
velocity response relative to the eddy forcing contributes to making direct observational verification of the present concepts difficult.

To summarize, on a local scale, topographic rectification is similar to traditional tidal rectification as discussed, for example, by Huthnance (1973) or by Loder (1980), except that along-isobath variability limits the mean flow magnitude and there is a spatial offset between eddy forcing and mean flow response. For more energetic fluctuating currents, eddy transport governs the mean flow. The present scaling argument for the magnitude of the mean flow can be criticized on a number of levels, including the use of the long-wave approximation. On the other hand, the underlying physics ought to be robust: the presence of a local mean vorticity gradient (in the form of a local depth gradient) should give rise to a spatial offset in response, just as, in a $\beta$-plane ocean, information spreads westward even in highly nonlinear contexts (e.g., Malanotte-Rizzoli and Holland, 1986; Aoki et al., 2009).

\section{Extensive, randomized topography}

One interesting aspect of the arguments leading to (27), (30) or (33) is that the time-mean flow development depends on the local depth gradient, but not on any measure of the larger scale, shelf-slope, gradient. If one is in the habit of thinking about topographic rectification in terms of asymmetric drag, as advocated by Brink (1986), HB or Samelson and Allen (1987), this is indeed astonishing, since this line of thinking requires the presence of systematic shelf-scale topography. In this sense, the present findings are much more attuned with the thinking of Holloway (1992) or Merryfield et al. (2001).

Two issues arising from Section 4 call for a different class of model runs, where there is no large-scale depth gradient, but where there is extensive random topography. First, there is the need to test the idea that the above parameterization, which is motivated by runs having shelf-slope topography, will still succeed in the absence of a larger-scale vorticity gradient. Second, there is a need to determine what the appropriate choice of topographic length scale, $L^{T}$, would be when the topographic variations are not simply sinusoids or combinations of a few sinusoids. Thus, four large-domain runs (Table 2) are carried out with randomized topographies described by (5). The topography is extensive enough that well-behaved correlation functions could be calculated and correlation length scales

$$
\Lambda^{\phi}=\int_{0}^{\infty} R^{\phi}(\xi) d \xi
$$

estimated, where $\phi$ is either $x$ or $y, R^{\phi}$ is the depth autocorrelation function in the $\phi$ direction, and $\xi$ is the spatial lag. In practice, (34) is evaluated numerically by integrating to large enough lags (typically $50 \mathrm{~km}$ ) that $\Lambda^{\phi}$ converges.

All four model runs (Table 2) give rise to time-mean flows with high topostrophy (0.67-0.72), i.e., there is a strong tendency for the time-mean flow to follow isobaths in the sense of topographic wave propagation (Fig. 6). Experiments show that the best time-mean flow parameterization is obtained by taking $L^{T}$ to be the minimum of $\Lambda^{x}$ or $\Lambda^{y}$ when the 


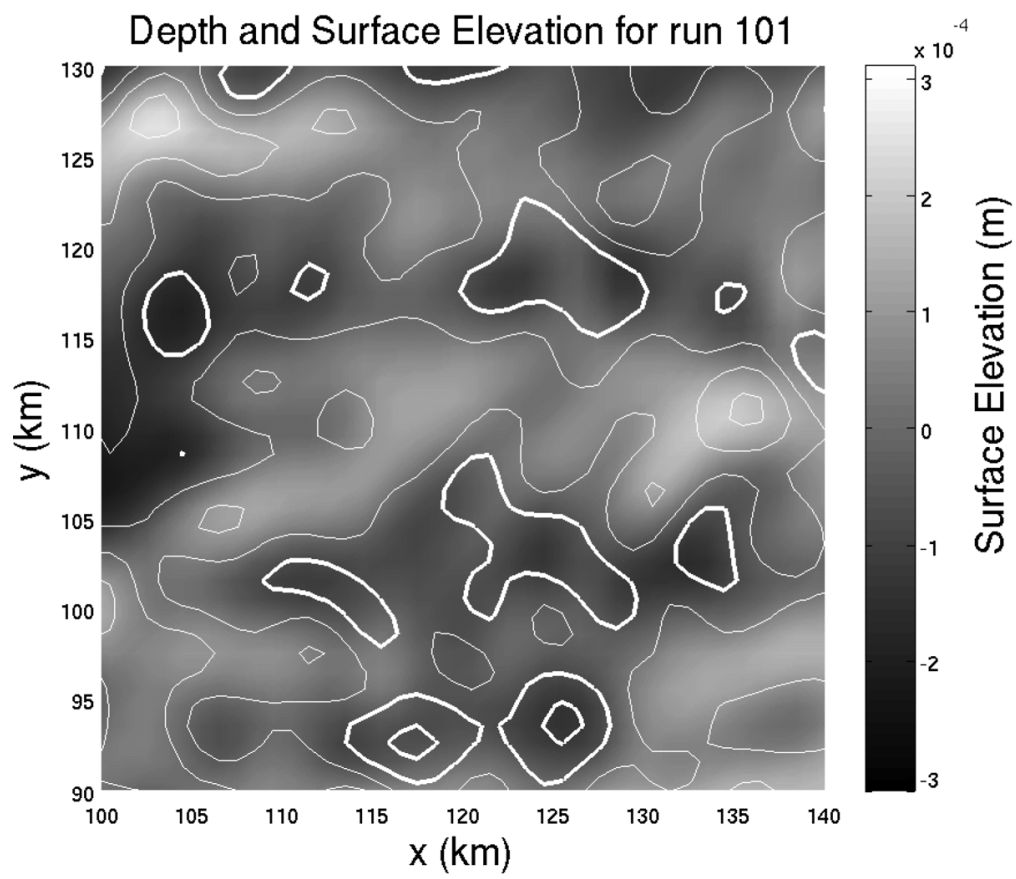

Figure 6. Sea-surface height (m) (shaded) and water depth (white contours). The contour interval for depth is $50 \mathrm{~m}$, and heavy contours are for depths $550,600, \ldots \mathrm{m}$. The mean water depth is $500 \mathrm{~m}$. Thus, shallow regions are outlined by thin contours, and deep regions by thick contours.

bottom topography is anisotropic. With this choice of length scale, using the weakest of (27), (30) and (33) for a time-mean flow estimate recovers $63 \%$ of the mean along-isobath velocity energy in the four runs combined (coefficients are adequately given by $a_{T}=0.45$, $a_{\omega}>10.0$ and $\left.a_{M}=0.7\right)$. Thus, the topographic wavelength of Section 4 is roughly equivalent to about $2 \Lambda^{\varphi} .99 .4 \%$ of the model grid points are represented by the variabletopography time-mean flow case (30), while none of the points obey the straight-isobath limit (27), and $0.6 \%$ of the points obey the strong-stirring limit (33). If the flow field is represented only by the curving-isobath case (30), the percentage of time-mean flow energy does not change. Some caution should be exercised in using the coefficients quoted here, since the model runs (Table 2) do not cover parameter space well.

The extensive domain runs demonstrate that the time-mean flow parameterization works well in the absence of a large-scale topographic gradient and where the topographic irregularity is random rather than periodic in character. This vindicates the idea that indeed the local depth gradient (i.e., "bumps") is the important factor, and that asymmetric drag associated with the overall bottom slope (i.e., shelf/slope topography) is not the appropriate way to think about the problem. It thus appears that the present parameterization is likely to be generally valid for a barotropic ocean. 


\section{Discussion}

The estimated localized mean flow parameterization is

$$
\overline{v^{\dagger}}=-\operatorname{sign}\left(f h_{\eta}\right) \min \left(\left|v^{T}\right|,\left|v^{\omega}\right|,\left|v^{M}\right|\right)
$$

where

$$
\begin{aligned}
& v^{T}=-a_{T} \operatorname{sign}(f) h_{\eta} S D(u \perp) L^{T} / h, \\
& v^{\omega}=-a_{\omega} f h_{\eta} S D(u \perp)^{2} /\left(h \omega^{2}\right), \\
& v^{M}=-a_{M} f h_{\eta} S D(u \perp)^{2} /(r \omega) .
\end{aligned}
$$

Zimmerman (1980) developed a tidal rectification theory with small-amplitude irregular topography that qualitatively anticipated some of the current findings, particularly in terms of the sense of the general mean flow orientation.

The formulation (35) might appear problematic in that each of the three parameterizations implies that $\overrightarrow{N_{0}^{\xi}} \geq 0$ everywhere because $h_{\eta} \geq 0$ by definition. Indeed, direct calculations of the actual (not parameterized) eddy transports show that this is usually the case, at least for along-isobath averages, i.e., that nearly always $\left\{\overline{N_{0}^{\bar{\xi}}}\right\}_{C} \geq 0$ and $r\left\{\overline{v_{1}^{\dagger}}\right\}_{C} \leq 0$ (see Section 4). A positive semidefinite $\overline{N_{0}^{\xi}}$ would be a problem if $\overline{N_{0}^{\xi}} \cong \overline{N_{0}^{y}}$ since (9) would be violated because the integrated cross-channel $(x)$ component of eddy along-channel $(y)$ momentum $\llbracket u^{\prime} v_{x}^{\prime} \rrbracket$ would be nonzero (cyclic conditions require that the $\llbracket v^{\prime} v_{y}^{\prime} \rrbracket$ term always integrates to zero). Yet, numerical model runs show that the integral $\llbracket u^{\prime} v_{x}^{\prime} \rrbracket$ is small compared to $r \llbracket v \rrbracket$ so that (9) is in fact valid. The resolution is that the alongshore $(y)$ component of momentum advection $\overline{N^{y}}$ actually has contributions from both the along- and cross-isobath advection components, viz.

$$
\overline{N^{y}}=\overline{N^{\xi}} \cos \theta+\overline{N^{\eta}} \sin \theta
$$

where the angle $\theta$ between an isobath and the alongshore $(y)$ direction is given by

$$
\cos \theta=\frac{h_{x}}{|\nabla h|} .
$$

Thus, if $\overline{N_{0}^{\xi}} \geq 0$ everywhere (as suggested by the scaling) and if $h_{x} \geq 0$ everywhere (i.e., depth increases monotonically offshore, as is the case for nearly all of the model runs in Table 1), then the $\overline{N_{0}^{\xi}}$ contribution to $\underline{N^{y}}$ is indeed $\geq 0$ everywhere. However, the spatial integral of (36a) can still be zero because the $\overline{N^{\eta}} \sin \theta$ term (associated with transport of cross-isobath momentum) contributes, and there can, in reality, be some negative, but not dominant, contribution due to $\overline{N^{\xi}} \cos \theta$ because, even though usually the along-isobath average $\left\{\overline{N_{0}^{\xi}}\right\}_{C} \geq 0$, the actual local value of $\overline{N_{0}^{\xi}}$ can sometimes be negative. The fact that (9) is found to hold despite the general tendency to have the along-isobath average $\left\{\overline{N_{0}^{\xi}}\right\}_{C} \geq 0$ requires that the compensating terms are always sufficient to assure that alongshore momentum is conserved. 
On a practical level, (35) gives an estimate for the expected strength of barotropic topographically rectified flow. However, at a given location, the present parameterization may not even predict the sign of the along-isobath flow correctly (Fig. 5). Because of the considerable scatter of the comparison between the mean flow parameterization vs. model results, the most convincing way to evaluate the parameterization relative to ocean observations would be to exploit large databases such as that compiled by Holloway (2008), and to anticipate a high degree of scatter (comparable to that in Fig. 5), just as Holloway (2008) found for observed topostrophy.

The numerically modeled mean flow, while roughly following isobaths, does not parallel them exactly, and $\Xi<1$ (e.g., Figs. 2, 6). This is not surprising because the idealized mean-flow vorticity equation (14) requires that the response of the mean pressure field be displaced and diffused relative to the eddy forcing. For example, the maximum response to eddy forcing ought to be offset in the direction of topographic wave propagation relative to the location of maximum eddy forcing. This is consistent with constraint (9), which requires that the area-averaged topographic stress $\llbracket h^{\prime} p_{y}^{\prime} \rrbracket$ balance damping $\rho r \llbracket v \rrbracket$ : if the geostrophic flow were exactly along isobaths, the total topographic stress would be zero. It is useful to compare this lack of exact topographic steering to the findings of Bretherton and Haidvogel (1976) and Salmon et al. (1976) who also found steering to be only approximate. In their cases, the larger scales of flow tended to parallel isobaths exactly, and, at shorter scales, the flow was much less structured by the topography.

The present approach (uniform forcing, $y$-varying topography) could readily be reformulated to have $y$-uniform topography and fluctuating currents that vary alongshore (Appendix B). A mean-flow scaling similar to (35) results where the topographic scale $L^{T}$ is replaced by an alongshore scale of the forcing $L^{W}$, although considerable caution is required to make sure that constraint (9) is obeyed. Thus, it appears that in the actual ocean, where both topography and forcing vary in space, the appropriate length scale ought to reflect both the topographic and forcing scales. Even if the fluctuating flow field were uniform, the present length scales are problematic, in that real topographies are rarely either smooth with imposed sinusoidal bumps (Section 4) or random with no large-scale depth trend (Section 5). Similarly, the choice of a single frequency $\omega$ presents problems in dealing with the actual ocean. Developing a practical measure of along-isobath scale, that accounts for current variability as well as realistically complex topography, is an important step toward making the present results useful on a practical basis, but it is beyond the scope of the present study.

It is worth asking what a typical magnitude for the barotropic rectified flow might be in practice. None of the model runs gives a maximum rectified flow stronger than about $0.4 \mathrm{~m} / \mathrm{sec}$, and that was for extremely strong forcing (run 318 with wind stress amplitude of $10 \mathrm{~N} / \mathrm{m}^{2}$ : stronger than a hurricane). More representative maximum modeled mean velocity magnitudes are about $0.05 \mathrm{~m} / \mathrm{sec}$ or less (Table 1). Consider the following representative values: $L^{T}=10-50 \mathrm{~km}, h_{\eta}=10^{-3}-10^{-2}, S D(u \perp)=0.1 \mathrm{~m} / \mathrm{sec}$ and 
$h=100 \mathrm{~m}$. Under these conditions, $v^{T}=0.002-0.1 \mathrm{~m} / \mathrm{sec}$. These are not strong velocities, even though they could integrate to substantial transports. Thus, at least to the extent that barotropic dynamics apply, topographic rectification would be marginal in accounting for some of the $O(0.1 \mathrm{~m} / \mathrm{sec})$ mean flows often observed in the coastal ocean.

Straight-isobath rectification as in Loder (1980) or (35c) can, under the same circumstances, suggest a very strong rectified flow $\left(0.1-1 \mathrm{~m} / \mathrm{sec}\right.$ for $f=1 \times 10^{-4} \mathrm{l} / \mathrm{sec}$ and $\omega=$ $\left.1 \times 10^{-5} 1 / \mathrm{sec}\right)$, but this would be associated with large, $O(100 \mathrm{~km})$, along-isobath displacements (24) that are substantially larger than common topographic scales. Thus, under these circumstances, (35b) would instead apply, and the mean flow would be a good deal weaker than suggested by (35c). The present results therefore appear to explain why it is that large mean flows consistent with (35c) are not found in conjunction with low-frequency flows in the ocean, even though the expression is clearly applicable to tidal rectification problems where the along-isobath particle excursion is relatively short and where the tides themselves have large spatial scales.

The present parameterization has value on another, more abstract, level. That is the reasoning (Section 4), that topographic rectification over irregular topography can apparently be explained simply as a variant of "tidal rectification" (e.g., Huthnance, 1973; Zimmerman, 1980) modified to account for finite along-isobath variability. The validity of (12) demonstrates that the overall (spatially integrated) balance is between along-isobath frictional drag and eddy momentum flux divergence. Along-isobath variability involves smoothing and a spatial offset of mean currents relative to eddy forcing, so that local eddy flux divergence does not obviously correlate with local mean flow. It thus appears that the present scalings for cross-isobath momentum transport divergence actually represent spatially smoothed results much more than actual local values.

It is intriguing to compare the present form for along-isobath flow over irregular topography (35b) to the inviscid quasigeostrophic form (e.g., Eby and Holloway, 1994)

$$
\overline{v^{\dagger}} \approx-f L^{N 2} h_{\eta} / h
$$

or to the inviscid finite-amplitude topography form of Merryfield et al. (2001)

$$
\overline{v^{\dagger}} \approx-f L^{N 2} h_{0}^{2} h_{\eta} / h^{3}
$$

where $L^{N}$ is their unknown constant. The second form collapses to (37a) when the actual depth is approximately the reference depth $h_{0}$ (as assumed in 20b). Either form of (37) arises from an unforced initial value problem that evolves adiabatically to a statistically steady state. Relation (35b) represents the physics of a case with ongoing forcing and dissipation. For all the different assumptions, (35b) and (37a) are identical if

$$
|f| L^{N 2}=a_{T} L^{T} S D(u \perp) .
$$

Similarly, the form (37a) can be matched against (35c) or (35d) to come up with other potential expressions for an equivalent $L^{N}$. The one common thread is that all of these forms involve the amplitude of the fluctuating currents. Given the differing physics, the 
significance of an equality like (38) is questionable, but having the "Neptune" mean current magnitude, hence scale $L^{N}$, be related to the eddy velocity seems intuitive, as might the idea that a topographic length scale ought to enter the problem. The parallelism of the expressions for mean flow strength (Eqs. 30 and 37), along with the approximate manner in which these mean flows follow isobaths, suggest a commonality among these different problems, even though the assumed roles of dissipation and forcing differ. If there is an equivalence, one needs to account for why inviscid numerical model runs would evolve to a state that occurs in the presence of dissipation.

The resolution of this conundrum may lie in the tidal rectification literature. Assuming along-isobath uniformity, Garreau and Maze (1992) and Visser (1994) demonstrated that a similar mean along-isobath flow develops in the seemingly rather different limits of small cross-isobath particle oscillations with dissipation, and of large-amplitude cross-isobath oscillations with no dissipation. Indeed, Visser (1994) puts the two limits into a common light of both being means to resolve the geostrophic degeneracy for a mean flow. It thus seems possible that a similar duality between frictional and finite-amplitude effects might help to clarify the relationship between the present results and those of the classic "Neptune" problem. An alternative examination of how the classical adiabatic theory relates to a real, dissipative ocean can be found in Holloway (2009).

The present analysis does not take into account the role of density stratification. Merryfield et al. (2001) provide an insightful discussion of the likely consequences of this effect, which include bottom intensification. It thus seems possible that stratification could allow rectified momentum to be more vertically concentrated, hence locally stronger than in a barotropic case. Analyzing stratified numerical model runs will need to account for a range of interesting additional effects, including near-bottom mixing and rectification of the bottom Ekman transport (e.g., Brink and Lentz, 2010). The stratified problem is currently under active consideration.

Acknowledgments. This work was sponsored by the Physical Oceanography Program at the National Science Foundation through grant OCE-0751731. Discussions with Karl Helfrich, Greg Holloway, Steven Lentz, and Jamie Pringle are all gratefully acknowledged.

\section{APPENDIX A}

\section{Evaluating the use of cyclic boundary conditions}

In a cyclic channel flow problem, there can be no alongshore sea level slope averaged over the length of the channel in the ROMS model. HB has been criticized because their cyclic geometry precludes an alongshore slope, which might occur over an extended section of the real ocean. The immediate questions are then (1) whether a noncyclic geometry would allow a net alongshore pressure gradient to build up, and (2) whether, in terms of the topographically rectified alongshore flow, it makes any difference. Twin numerical model experiments are used to answer this question.

The obvious comparison to make would be between a strictly cyclic (re-entrant in the 
alongshore direction) topography (with, say, sinusoidal corrugations alongshore over the entire channel length) and a comparable problem with open boundary conditions at each end. The cyclic geometry essentially represents an infinitely long channel that admits no alongshore-mean alongshore pressure gradient, and the results should be the same at any alongshore location (as long as one is at the same spatial phase of the alongshore topographic corrugation). The second case, with open boundary conditions, will not prevent an alongshore pressure gradient from evolving.

This twin approach has one "apples to oranges" aspect to it, and that is because, for the open case, the upwave portion (meaning the end from which long topographic Rossby waves propagate) of the model domain (i.e., large $y$, assuming a shelf-slope geometry as in Figure 1) will experience end effects over a distance set by long coastal-trapped wave frictional decay. This scale is $L_{F}=O\left(T_{F n} c_{n}\right)$, where $c_{n}$ is the phase speed of mode $n$, and $T_{F n}$ is a frictional decay time for that mode. Because the phase speed decreases with increasing $n$, it is sufficient to consider the gravest, $n=1$ wave (the $n=0$ or barotropic Kelvin wave, is not expected to contribute substantially to the velocity field because of its very large cross-shelf scales). In contrast, coastal-trapped waves (even in the barotropic case) do not carry information efficiently upwave from the downwave ( $y=0)$ boundary, so the area near the downwave end that "feels" end effects is much shorter than near the upwave end. At distances from the ends greater than these decay scales, the open channel ought to be comparable to the strictly cyclic case unless a net alongshore pressure gradient is important in the problem.

Thus, a well-designed numerical experiment with open boundary conditions would have a channel long enough that the end effects are not felt over a substantial part of its length. This requires $L_{B} \gg L_{F}$, where $L_{B}$ is the open-ended channel length. Only a portion far (compared to $L_{F}$ ) from the upwave (large $y$ ) boundary would be compared against cyclic results. Unfortunately, an open numerical model configuration could not be found that both conserved volume and allowed information to pass smoothly out of the domain. An alternative configuration with a very long re-entrant channel is thus used (Fig. A1). Specifically, the irregular bottom topography covers an alongshore span $L_{B} \gg L_{F}$, and, in addition, a very long (length $L_{S}$ ) region with a smooth bottom is inserted into the domain. The key assumption is that $L_{S}$ is so great (relative to $L_{F}$ ) that no re-entrant "memory" of the bumpy region would be carried back into the regime from the upwave end. Models with this general configuration are compared to runs with the same length domain, but having topographic corrugations over the entire length.

Seven model comparisons are run, with different forcing period, bottom friction, forcing amplitude, corrugation amplitude, corrugation wavelength, and Coriolis parameter. The wave speed, hence decay distance, is kept small by keeping the flat, deep ocean depth $h_{M}$ at $125 \mathrm{~m}$. Shelf wave parameters are computed using the barotropic code in Brink and Chapman (1987): usually, $L_{F} \approx 210 \mathrm{~km}$ and $c_{1}=2.1 \mathrm{~m} / \mathrm{sec}$ (except for two cases of $L_{F} \approx 110 \mathrm{~km}$ and one case with $c_{1}=1.1 \mathrm{~m} / \mathrm{sec}$ ). Thus, the choices $L_{B}=960 \mathrm{~km}$ and $L_{S}=3500 \mathrm{~km}$ assure that the design criteria are met tolerably. All comparison model runs 


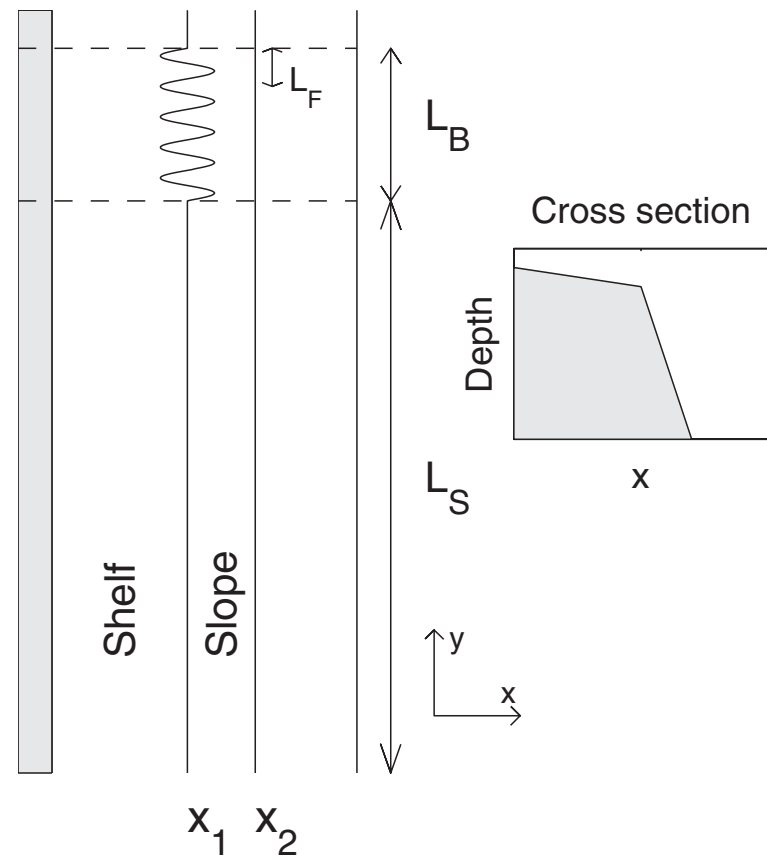

Figure A1. Definition sketch for the twin experiments evaluating cyclic geometry.

are carried out for 58 days, and the comparisons are made using only the last forcing period.

Results from a representative model run, using topographic wavelength of $60 \mathrm{~km}$ in the corrugated section, are shown in Figures A2 and A3. A sinusoidal (in time) alongshore wind stress of amplitude $0.1 \mathrm{~N} / \mathrm{m}^{2}$ and period 4 days is applied over the entire domain. The corrugation amplitude at the shelfbreak $(x=53 \mathrm{~km})$ is $21 \mathrm{~m}$, while the bottom resistance coefficient is $r=5 \times 10^{-4} \mathrm{~m} / \mathrm{sec}$. Both pressure and alongshore velocity are smoothed for plotting purposes by averaging alongshore over one-wavelength $(60 \mathrm{~km})$ bins. The mean pressure field in the corrugated region (Fig. A2) builds up rapidly over roughly the upwave $400 \mathrm{~km}$ (compare to $L_{F} \approx 210 \mathrm{~km}$ ), and then adjusts to a nearly constant alongshore pressure gradient for $4200>y>3800 \mathrm{~km}$. (Note that the axes on Figure A2 are distorted by about a factor of 14 , so the apparent alongshore pressure gradient is greatly exaggerated.) The shelf-slope pressure always decreases in the offshore direction over the topography, since the residual mean alongshore flow is in approximately geostrophic balance (cross-shelf flow, however, has a substantial ageostrophic component).

The pressure distribution in Figure A2 shows that there is a closed horizontal circulation cell in the $y$ range where the bottom is corrugated. Over most of the shelf-slope region where the bottom is irregular, mean flow is downwave, but in the "deep ocean" $(x>72 \mathrm{~km})$ where the bottom is flat, mean flow is upwave, closing the cell. The onshore 

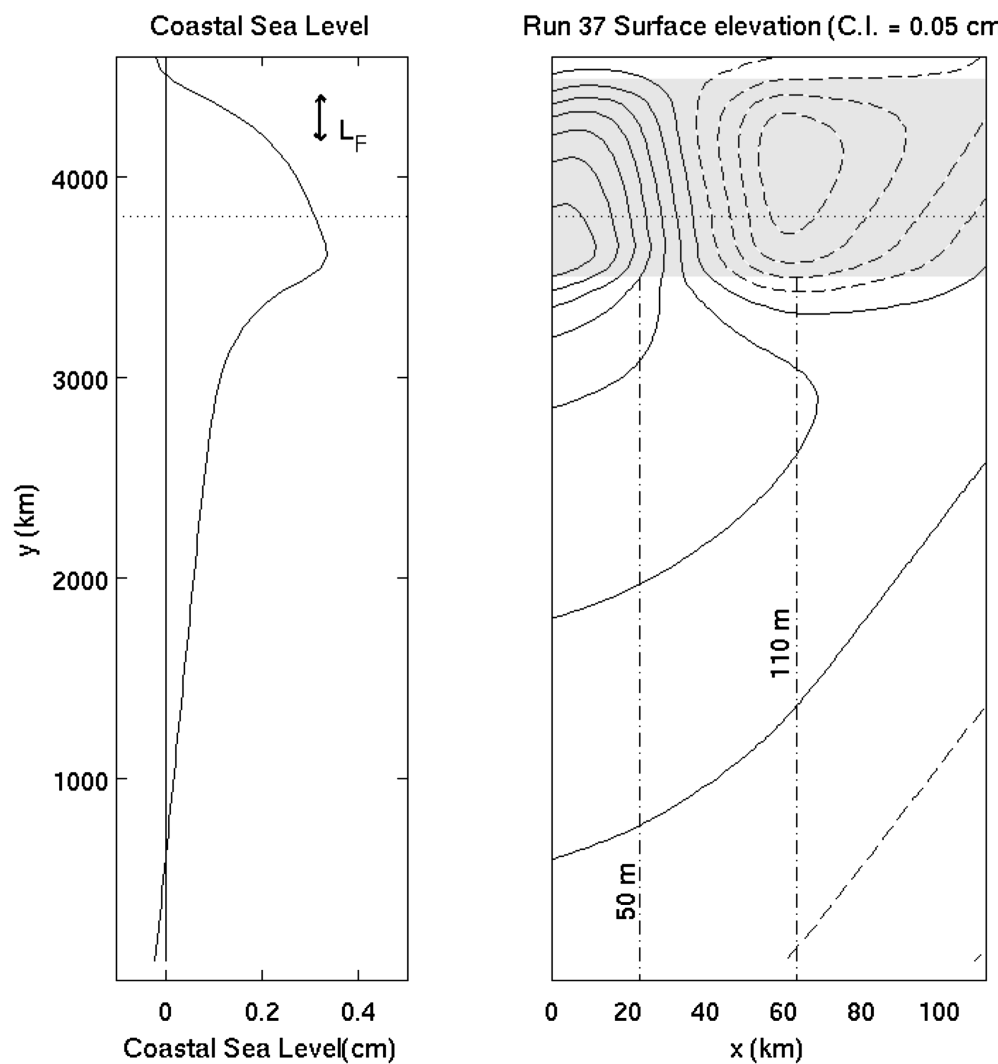

Figure A2. Smoothed (over corrugation scales) sea-surface height, averaged over the last four-day period, for model run 37. The bottom is corrugated only within the shaded region (amplitude at $x=$ $53 \mathrm{~km}$ is $21 \mathrm{~m}$, and the wavelength is $60 \mathrm{~km}$ ). Isobaths are shown only where the bottom is smooth. The location for comparing mean alongshore velocity is shown by a dotted line. Left panel. Sea level at the coast. The frictional decay length scale $L_{F}$ is shown for comparison. Right Panel. Plan view. Zero and positive height are shown as solid contours, and negative elevations are shown as dashed contours. The contour interval is $0.05 \mathrm{~cm}$.

flow occurs at the upwave end of the corrugated area $(y>4300 \mathrm{~km})$ while the offshore flow is distributed over about $y=2800-4300 \mathrm{~km}$, peaking near $y=3800 \mathrm{~km}$ (i.e. near the downwave edge of the corrugations at $y=3500 \mathrm{~km}$ ).

Smoothed mean alongshore velocity is sampled at $y=3800 \mathrm{~km}$, near the downwave edge of the corrugated region, and compared to results from the twin strictly cyclic model (Fig. A3). In the cyclic case, all smoothed flow is strictly alongshore, and the isobars parallel the coast. Agreement for $\bar{v}$ between the two runs over the shelf-slope region $(x<72 \mathrm{~km})$ is reasonably good. The main difference between the two velocity profiles occurs in the flat-bottom offshore region where, for the strictly cyclic case, flow is 


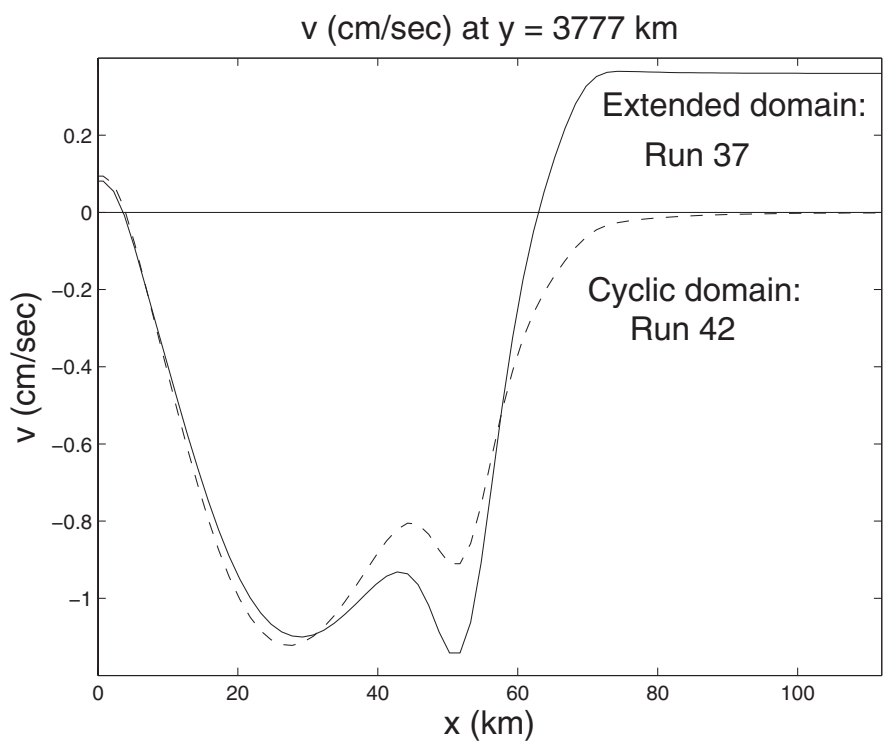

Figure A3. Smoothed time mean alongshore velocity at $y=3800 \mathrm{~km}$ (dotted line in Fig. A2). Solid line: run 37 (as in Fig. A2). Dashed line: results from a strictly cyclic, but otherwise identical run (number 42).

downwave and generally very weak, but the noncyclic case has an upwave (toward positive $y$ ) return flow here. This example is typical of all of the comparisons made.

In conclusion, it appears that strict alongshore periodicity does not introduce undesirable effects in the numerical solution. In the interior of the corrugated region, a weak (compared to the cross-shelf gradient) alongshore pressure gradient does form in the "open" case, but it is largely balanced by the cross-shelf flow of the circulation cell, and it does not seem to have a substantial effect (compared to the strictly cyclic runs) on the net downwave flow in places where the bottom is not flat.

\section{APPENDIX B}

\section{Spatially non-uniform forcing over uniform topography}

It appears that the problem with straight isobaths and alongshore varying wind stress ought to be analogous to the problem with uniform wind stress and alongshore topographic variations. In fact, the scaling analysis of Section 4 carries through exactly, except that (1) it now remains in $(x, y)$ coordinates, (2) the alongshore scale is now the alongshore wavelength of the wind stress $L^{W}$ rather than the topographic wavelength $L^{T}$, and (3) the constraint (9) must be accounted for because the topographic stress term vanishes.

To test whether this scaling applies to the variable-wind problem, a set of 55 barotropic numerical model runs was carried out. The runs were forced by a fluctuating cross-shelf wind stress 

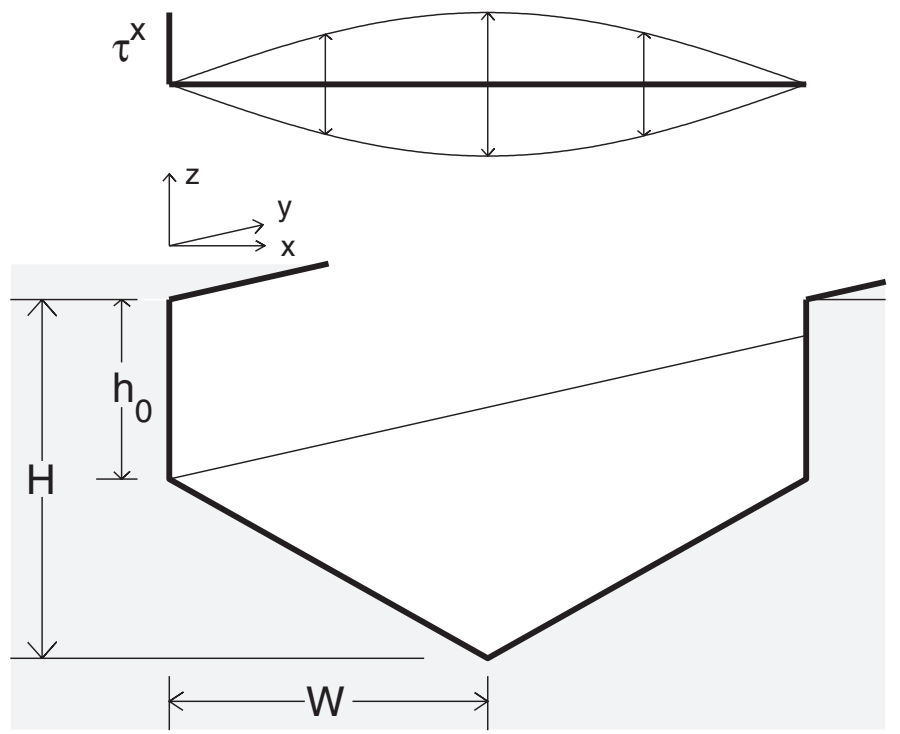

Figure A4. Definition sketch for numerical model runs with straight isobaths, and spatially varying wind stress.

$$
\tau^{x}=T_{0} \sin [\pi x /(2 W)] \cos (2 \pi y / \lambda) \sin (\omega t)
$$

and the geometry is described by Figure A4. The wind forcing (A1) is used because it results in a mean flow without any strong lateral boundary layers. The symmetric (about $x=W$ ) geometry is motivated by (9): this special geometry allows the no-mean-stress condition to be met without severely constraining the flow. For all numerical model runs, $f=1 \times 10^{-4} \mathrm{l} / \mathrm{sec}, W=40 \mathrm{~km}$ and $H=200 \mathrm{~m}$. The space of nondimensional numbers is then explored by varying forcing amplitude $T_{0}$, water depth at the coast $h_{0}$, bottom friction $r$ and the wind's wavelength $\lambda$ and frequency $\omega$.

As in the case with irregular topography, many of the runs result in frequency multiplication or chaotic solutions. A regime diagram qualitatively similar to Figure 4 then describes the distribution of chaotic runs. A set of 31 nonchaotic runs is used to evaluate the coefficients in (35) for this problem, and this fit accounts for $63 \%$ of the energy in the mean along-isobath flow for these runs. The coefficients of the fit are then $\left(a_{T}^{W}, a_{\omega}^{W}, a_{M}^{W}\right)=$ $(0.21,0.34,0.16)$, not dissimilar to the values for variable topography.

\section{REFERENCES}

Alvarez, A., E. Hernandez-Garcia and J. Tintore. 1997. Noise-sustained currents in quasigeostrophic turbulence over topography. Physica A, 247, 312-326.

Aoki, K., A. Kubokawa, H. Sasaki and Y. Sasai. 2009. Mid-latitude baroclinic Rossby wave in a high-resolution OGCM Simulation. J. Phys. Oceanogr., 39, 2264-2279.

Bretherton, F. P. and D. B. Haidvogel. 1976. Two-dimensional turbulence over topography. J. Fluid Mech., 78, 129-154. 
Brink, K. H. 1986. Topographic drag due to barotropic flow over the continental shelf and slope. J. Phys. Oceanogr., 16, 2150-2158.

Brink, K. H. and D. C. Chapman. 1987. Programs for computing properties of coastal-trapped waves and wind-driven motions over the continental shelf and slope, 2nd ed., Woods Hole Oceanographic Institution Technical Report WHOI-87-24, $119+$ iii pp.

Brink, K. H. and S. J. Lentz. 2010. Buoyancy arrest and bottom Ekman transport: Part II, oscillating flow. J. Phys. Oceanogr., 40, 636-655.

Carnevale, G. F. and J. S. Frederiksen. 1987. Nonlinear stability and statistical mechanics of flow over topography. J. Fluid Mech., 175, 157-181.

Chen, C. and R. C. Beardsley. 1995. A numerical study of stratified tidal rectification over finite-amplitude banks. Part I symmetric banks. J. Phys. Oceanogr., 25, 2090-2110.

Csanady, G. T. 1978. The arrested topographic wave. J. Phys. Oceanogr., 8, 47-62.

Cummins, P. F. 2000. Remarks on potential vorticity mixing over topography and momentum conservation. Deep-Sea Res. I, 47, 737-743.

Eby, M. and G. Holloway. 1994. Sensitivity of a large-scale ocean model to parameterization of topographic stress. J. Phys. Oceanogr., 24, 2577-2588.

Garreau, P. and R. Maze. 1992. Tidal rectification and mass transport over a shelf break: A barotropic frictionless model. J. Phys. Oceanogr., 22, 719-731.

Gill, A. E. 1982. Atmosphere-Ocean Dynamics, Academic Press, NY, 662 pp.

Gill, A. E. and E. H. Schumann. 1974. The generation of long shelf waves by the wind. J. Phys. Oceanogr., 4, 83-90.

Haidvogel, D. B. and K. H. Brink. 1986. Mean currents driven by topographic drag over the continental shelf and slope. J. Phys. Oceanogr., 16, 2159-2171.

Herring, J. R. 1977. On the statistical theory of two-dimensional topographic turbulence. J. Atmos. Res., 34, 1731-1750.

Holloway, G. 1978. A spectral theory of barotropic motion above irregular topography. J. Phys. Oceanogr., 8, 414-427.

1987. Systematic forcing of large-scale geophysical flows by eddy-topography interaction. J. Fluid Mech., 184, 463-476.

1992. Representing topographic stress for large-scale ocean models. J. Phys. Oceanogr., 22, $1033-1046$.

2008. Observing global ocean topostrophy. J. Geophys. Res., 113, C07054, doi:10.1029/ 2007JC004635.

-2009. Entropic forces in geophysical fluid dynamics. Entropy, 11, 360-383.

Holloway, G. and T. Sou. 1996. Measuring the skill of a topographic stress parameterization in a large-scale ocean model. J. Phys. Oceanogr., 26, 1088-1092.

Huthnance, J. M. 1973. Tidal current asymmetries over the Norfolk Sandbanks. Estuar. Coastal Mar. Sci., 1, 89-99.

LaCasce, J. H., O. A. Nøst and P. E. Isachsen. 2008. Asymmetry of free circulation in closed gyres. J. Phys. Oceanogr., 38, 517-526.

Loder, J. W. 1980. Topographic rectification of tidal currents on the sides of Georges Bank. J. Phys. Oceanogr., 10, 1399-1416.

Maas, L. R. M. and J. T. F. Zimmerman. 1989. Tide-topography interactions in a stratified shelf sea II. Bottom trapped internal tides and baroclinic residual currents. Geophys. Astrophys. Fluid Dyn., 45, 37-69.

Maas, L. R. M., J. T. F. Zimmerman and N. M. Temme. 1987. On the exact shape of the horizontal profile of a topographically rectified tidal flow. Geophys. Astrophys. Fluid Dyn., 38, 105-130.

Malanotte-Rizzoli, P. and W. Holland. 1986. Data constraints applied to models of the ocean general circulation. Part I: The steady case. J. Phys. Oceanogr., 16, 1665-1682. 
Merryfield, W. J. 1998. Effects of stratification on quasi-geostrophic inviscid equilibria. J. Fluid Mech., 354, 345-356.

Merryfield, W. J., P. F. Cummins and G. Holloway. 2001. Equilibrium statistical mechanics of barotropic flow over finite topography. J. Phys. Oceanogr., 31, 1880-1890.

Ou, H. W. 1999. A model of tidal rectification by potential vorticity mixing. Part I: Homogeneous ocean. J. Phys. Oceanogr., 29, 821-827.

Perenne, N., A. Pichon and P. Huet. 2000. A numerical study of tidal rectification. Cont. Shelf Res., 20, 37-68.

Robinson, I. S. 1981. Tidal vorticity and residual circulation. Deep-Sea Res. A., 28, 195-212.

Salmon, R., G. Holloway and M. C. Hendershott. 1976. The equilibrium statistical mechanics of simple quasi-geostrophic models. J. Fluid Mech., 75, 691-703.

Samelson, R. M. and J. S. Allen. 1987. Quasi-geostrophic topographically generated mean flow over the continental margin. J. Phys. Oceanogr., 17, 2043-2094.

Shchepetkin, A. F. and J. C. McWilliams. 2005. The regional oceanic modeling system (ROMS): A split-explicit, free-surface, topography-following-coordinate oceanic model. Ocean Model., 9, 347-404.

Stern, M. E. and C. Y. Shen. 1976. Displacement and rectification of planetary fluids. Geophys. Fluid Dyn., 7, 81-118.

Treguier, A. M. and J. C. McWilliams. 1990. Topographic influences on wind-driven, stratified flow in a $\beta$-plane channel: An idealized model for the Antarctic Circumpolar Current. J. Phys. Oceanogr., 20, 321-343.

Veronis, G. 1963. An analysis of wind-driven ocean circulation with a limited number of Fourier components. J. Atmos. Res., 20, 577-593.

Visbeck, M., J. Marshall and H. Jones. 1996. Dynamics of isolated convective regions in the ocean. J. Phys. Oceanogr., 26, 1721-1734.

Visser, A. W. 1994. On tidal rectification, friction and geostrophic degeneracy. J. Phys. Oceanogr., 24, 2196-2200.

Wright, D. G. and J. W. Loder. 1985. A depth-dependent study of the topographic rectification of tidal currents. Geophys. Astrophys. Fluid Dyn., 31, 169-220.

Zimmerman, J. T. F. 1978. Topographic generation of residual circulation by oscillatory (tidal) currents. Geophys. Astrophys. Fluid Dyn., 11, 35-47.

1980. Vorticity transfer by tidal currents over irregular topography. J. Mar. Res., 38, 601-630.

Received: 29 January, 2010; revised: 10 September, 2010. 